\title{
On the Static Interaction of Fluid and Gas Loaded Multi-Chamber Systems in Large Deformation Finite Element Analysis
}

\author{
M. Haßler, K. Schweizerhof \\ Universität Karlsruhe, Institut für Mechanik
}

2007

Institut für Mechanik

Kaiserstr. 12, Geb. 20.30

76128 Karlsruhe

Tel.: +49 (0) 721/ 608-2071

Fax: +49 (0) 721/ 608-7990

E-Mail: ifm@uni-karlsruhe.de

www.ifm.uni-karlsruhe.de 


\title{
On the Static Interaction of Fluid and Gas Loaded Multi-Chamber Systems in Large Deformation Finite Element Analysis
}

\author{
M. Haßler, K. Schweizerhof
}

25. Januar 2007

\begin{abstract}
Completing previous studies [5], [6], [18], [19] and [20] on the deformation dependence of gas or fluid loadings with the assumption of finite gas and fluid volumes, the current contribution focuses on the influence of modifications of the size and shape of a finite structure filled with combinations of gas and incompressible or compressible heavy fluid. In contrast to the previous contributions [5], [18], [19] and [20], where different finite element formulations for some specific load cases were derived, this derivation provides a general formulation of a multi chamber system filled with gas and additional compressible heavy fluid. By introducing two stiffness parameters it is possible to reduce the formulation to the pure pneumatic or hydrostatic load cases described in earlier works. Further on it will be shown how to manage the modification of the load cases during the loading process. The linearization of the corresponding virtual work expression, necessary for a Newton-type solution algorithm, leads to additional terms for the volume dependence [6], [19], [20] and the expected fully symmetric stiffness matrix. The discretization of the solid structures with finite elements leads to standard stiffness matrix forms for the structures plus the so-called load stiffness matrices [26] and several dyadic rank updates - depending on the type of filling - for each filled structure part, assuming that loaded surface segments are identical with the element surfaces. A further focus is on a specific solution scheme exploiting the dyadic rank update structure. Some numerical examples show the effectiveness of the approach and the necessity to take the corresponding terms in the variational expression and in the linearization into account.
\end{abstract}

Keywords: hydrostatic fluid loading, gas loading, volume dependence, large deformations, finite elements, multi chamber problems

\section{Introduction}

This contribution tries to provide a realistic and general description of the interaction of arbitrarily combined fluid and/or gas loaded or filled multi-chamber systems undergoing large static or quasi-static deformations. Unlike in conventional FE simulations, where an internal pressure invariant to the structural deformations and thus to the volume change is assumed this derivation of the fluid structure interaction takes into account that the inner state variables, namely fluid level, fluid density and gas pressure - fluid and gas both in clearly described volumes - depend on the shape and the volume of the surrounding surfaces. Further on in this contribution also the normal change effects of displacement dependent pressure loads in large deformation analyses, which have already been described 
in [24], [26] are considered. Thus it is possible to derive an analytical formulation of the fluid/gas effects only described by surface integrals, which makes any FE discretization of the fluid or the gas redundant. Early investigations on pure gas loaded membrane structures considering both the volume change of the gas and follower force effects of the pressure can be found in [5], [6]. At this point we have to refer to van Dijk [8] who already discussed partially fluid and gas filled containers, however, the effects of the nonuniform hydrostatic pressure were neglected in comparison to the internal gas pressure. Consequently the derivations were restricted to small fluid and gas filled structures with small deformations, e.g. drinking bottles, but extensions are needed for larger containments as oil or water tanks, where the hydrostatic pressure dominates. In addition structures undergoing large deformations, such as membranes, for which the water depth is modified due to deformations, were not considered in [8], as gravity effects have been neglected. All contributions in fluid-structure interaction considering compressible fluids and fluids in gravity field with a free fluid surface [1], [2], [22], [23] discretize the fluids by finite elements. To date a meshless formulation of an arbitrary combination of gas and fluid loaded multi chamber systems in a gravity field was not proposed (i.e. not known to the authors). In earlier contributions of the authors group gas supported shell structures [19] and shell structures loaded with incompressible fluids [20] as well as structures fully filled with compressible fluids [18] have already been discussed in the context of finite deformations. Now these results are merged to a generalized algorithm for closed chambers containing any combination of gas and fluid in order to simulate arbitrary types of static loading and emptying of closed strongly deforming structures containing fluid and/or gas.

First a consistent derivation of a containment filled with both gas and a compressible heavy fluid will be briefly presented via their virtual work expressions. In a further step the obtained variation of the gas/fluid potential has to be linearized in order to provide for a Newton-type solution algorithm. A particular focus of this work will also be on the solution process: The dyadic extended equation system can be solved very efficiently in the context of direct solvers by a sequential application of the Sherman-Morrison formula, for which a general vectorized algorithm is presented. The numerical examples contain large deformation analyses of fluid and gas filled shell type structures with rather thin flexible walls and illustrate the performance of the derived algorithms.

\section{Governing Equations}

The general problem (see figure 2.1) is described by its properties of the potential energy $\Pi$, which consists of the - here for simplicity - elastic potential $\Pi^{e l}$ of the surrounding structure of the containment, the potential $\Pi^{f s i}$ of the internal fluid and gas and the work $W^{e x}$ of the external forces. For equilibrium the variation of the total potential energy then vanishes identically. We have to note that all following derivations concerning the fluid/gas expressions are also valid for structural inelastic or viscous behavior.

\subsection{Virtual Work Expression}

The variation $\delta \Pi$ of the total potential energy is given by the variation of its parts:

$$
\delta \Pi=\delta \Pi^{e l}+\delta \Pi^{f s i}-\delta W^{e x}=0
$$

For the virtual elastic energy $\delta \Pi^{e l}$ and the virtual work $\delta W^{e x}$ of the standard external loading, such as e.g. dead loading, we refer to standard FE-text books [27], [29]. In the 


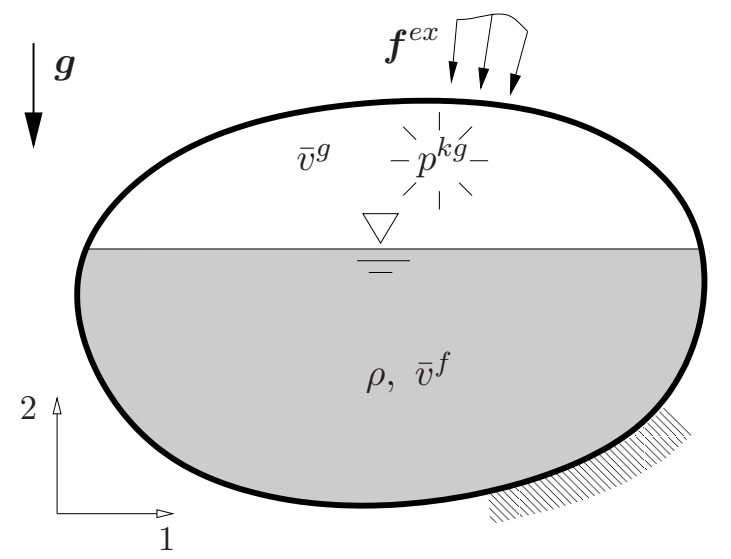

Fig. 2.1: Elastic shell structure filled with heavy fluid and gas

following the focus is only on the variation of the potential of the fluid-structure-interaction (fsi) part and the gas or fluid loading

$$
\delta \Pi^{f s i}=\delta V^{f}-\delta W^{g}-\delta W^{f},
$$

which consists of the variation of the gravity potential $\delta V^{f}$ of the heavy fluid, of the virtual work $\delta W^{g}$ resulting from the gas compression and of the virtual work $\delta W^{f}$ resulting from the fluid compression. As already described in [18], the variation of the potential

$$
\begin{aligned}
\delta V^{f} & =\delta\left(-\rho \boldsymbol{g} \cdot \overline{\boldsymbol{s}}^{f}\right) \\
& =-\delta \rho \boldsymbol{g} \cdot \overline{\boldsymbol{s}}^{f}-\rho \boldsymbol{g} \cdot \delta \overline{\boldsymbol{s}}^{f}
\end{aligned}
$$

due to acceleration of gravity $\boldsymbol{g}$ can be written in terms of the fluid density $\rho$ and its first order volume moment $\overline{\boldsymbol{s}}^{f}$, which will be given in detail later. The virtual work expressions of the gas and fluid volume compression (see also [28]) are

$$
\delta W^{g}=p^{k g} \delta \bar{v}^{g}
$$

and

$$
\delta W^{f}=p^{k f} \delta \bar{v}^{f} .
$$

The equations contain the gas and fluid volume changes $\delta \bar{v}^{g}$ and $\delta \bar{v}^{f}$ and the volume dependent pressures $p^{k g}$ for the gas and $p^{k f}$ for the fluid.

\subsection{Constitutive Equations}

To obtain the remaining terms in the variation of the $f s i$-potential $\delta \Pi^{f s i}$ mass conservation and the constitutive equations have to be considered.

Mass conservation

Due to mass conservation the density change $\delta \rho$ of a fluid is correlated with the volume change $\delta \bar{v}^{f}$ of a fluid:

$$
\delta \rho=-\frac{\rho}{\bar{v}^{f}} \delta \bar{v}^{f}
$$


Here we assume that the density change of a fluid in a gravity field is uniform and independent of the height of the fluid volume. In general an exponential density increase with increasing fluid depth $h$ is assumed (see [18])

$$
\frac{\Delta \rho}{\rho_{0}}=\exp \left(\frac{|\boldsymbol{g}| \rho_{0}}{K} h-1\right) \quad(\text { with bulk modulus } K) \text {, }
$$

which shows that for technical applications the assumption (2.6) is sufficient even with fairly large fluid heights $h$.

\section{Adiabatic state equation}

The adiabatic state equation provides us with the gas pressure in the current state

$$
p^{k g}=p_{0}^{k g}\left(\frac{\bar{v}_{0}^{g}}{\bar{v}^{g}}\right)^{\kappa}
$$

with $p_{0}^{k g}$ and $\bar{v}_{0}^{g}$ denoting the pressure and the volume of the gas in the initial state and $\bar{v}^{g}$ its current volume. For a two-atomic gas we can set the isentropic exponent to $\kappa=1.4$ [4]. With equation (2.4) it is assumed that we have perfectly isolated control volumes with no loss of heat and with no production of entropy due to volume compression. At this point we have to note that temperature effects could be included in the equations following standard measures. Setting $\kappa=1.0$ leads to Boyle's law describing the isothermal behavior of an ideal gas.

Hooke's law

With Hooke's law the dependence of the inner fluid pressure on a volume change is given:

$$
p^{k f}=-\frac{\bar{v}^{f}-\bar{v}_{0}^{f}}{\bar{v}_{0}^{f}} K .
$$

$\bar{v}_{0}^{f}$ and $\bar{v}^{f}$ represent a reference fluid volume and the current one and $K$ denotes the bulk modulus of the fluid.

Identity of the pressures due to volume compression

This final equation is only needed for chambers partially filled with gas and fluid with continuous gas and fluid subvolumes, see figure 2.2a). At the common interface of fluid and gas the remaining pressures $p^{k g}$ and $p^{k f}$ therefore in the case of single gas and fluid subvolumes must satisfy the equilibrium condition

$$
p^{k g}=p^{k f}=p^{k} .
$$

A possible separation of control volumes during the deformation process, see figure $2.2 \mathrm{~b}$ ), is not included in this equation, although it is in principle directly possible to include such separation processes. Thus in the following only chambers with one fluid and one gas part are considered. To avoid any misunderstanding concerning the origin of the volume compression terms, despite their identity the different notations $p^{k g}$ and $p^{k f}$ will be used further on.

\subsection{Boundary Integral Representation of the Geometry}

In section 2.1 the variation of the fluid potential was given in terms of the variables $\delta \bar{v}^{g}$, $\delta \bar{v}^{f}$ and $\delta \overline{\boldsymbol{s}}$ describing the geometrical attributes of the fluid and gas. Consequently the second step will now be the representation of these geometrical quantities by boundary integrals, enabling an analytical "meshfree" description of the enclosed gas and heavy fluid 
a) gas volume continuous

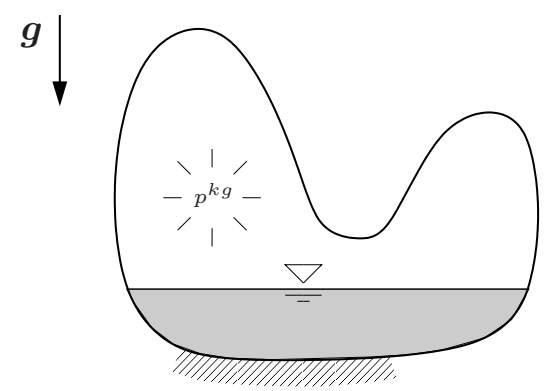

b) gas volume discontinuous

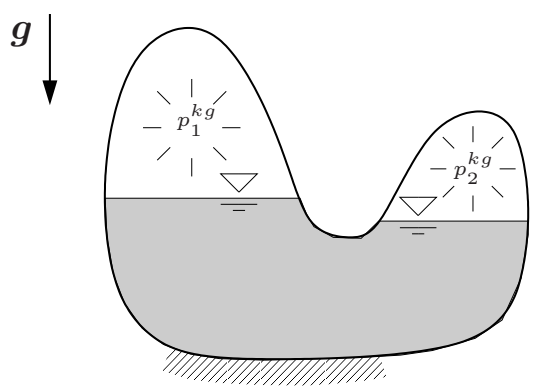

Fig. 2.2: a) Considered and b) not treated configurations of gas and fluid filled chambers

via their surrounding wetted surface, e.g. later discretized by FE or BE. Sectioning both control volumes $\bar{v}^{g}$ and $\bar{v}^{f}$ a three dimensional projection of the geometry for the virtual gas and fluid volumes yields, see figure 2.3,

$$
\begin{aligned}
& \delta \bar{v}^{g}=\delta v^{g}-\delta v^{o}=\int_{\Gamma^{g}} \overline{\boldsymbol{n}}^{g} \cdot \delta \boldsymbol{u}^{g} d \Gamma-\int_{\Gamma^{o}} \overline{\boldsymbol{n}}^{o} \cdot \delta \boldsymbol{u}^{o} d \Gamma, \\
& \delta \bar{v}^{f}=\delta v^{f}+\delta v^{o}=\int_{\Gamma^{f}} \overline{\boldsymbol{n}}^{f} \cdot \delta \boldsymbol{u}^{f} d \Gamma+\int_{\Gamma^{o}} \overline{\boldsymbol{n}}^{o} \cdot \delta \boldsymbol{u}^{o} d \Gamma,
\end{aligned}
$$

see also [6],[19] and [21]. According to [7] with the surface integral formulation of a first order volume moment

$$
\boldsymbol{s}=\frac{1}{4} \int_{\Gamma}\left(\overline{\boldsymbol{n}}^{f} \cdot \boldsymbol{x}^{f}\right) \boldsymbol{x}^{f} d \Gamma
$$

for the virtual first order volume moment of the heavy fluid, necessary for the gravity effect, we can write

$$
\delta \overline{\boldsymbol{s}}^{f}=\delta \boldsymbol{s}^{f}+\delta \boldsymbol{s}^{o}=\int_{\Gamma^{f}}\left(\overline{\boldsymbol{n}}^{f} \cdot \delta \boldsymbol{u}^{f}\right) \boldsymbol{x}^{f} d \Gamma+\int_{\Gamma^{o}}\left(\overline{\boldsymbol{n}}^{o} \cdot \delta \boldsymbol{u}^{o}\right) \boldsymbol{x}^{o} d \Gamma .
$$

Because the fluid level $\boldsymbol{x}^{o}$ is independent of the quantities describing the surface integral, it can be shifted out of the integral:

$$
\delta \boldsymbol{s}^{o}=\int_{\Gamma^{o}} \boldsymbol{n}^{o} \cdot \delta \boldsymbol{u}^{o} d \Gamma \boldsymbol{x}^{o}=\delta v^{o} \boldsymbol{x}^{o}
$$

The geometrical quantities in equations (2.11) and (2.14) describe the current configuration, as equilibrium has to be satisfied in the current configuration. In the integrals $\boldsymbol{x}$ denotes the position vector to an arbitrary point on the shell surface, $\boldsymbol{x}^{o}$ denotes the location of the free fluid surface and $\overline{\boldsymbol{n}}$ denotes the normalized normal vector of a discrete area element

$$
d \Gamma=\left|\boldsymbol{x}_{, \xi} \times \boldsymbol{x}_{, \eta}\right| d \xi d \eta=|\boldsymbol{n}| d \xi d \eta
$$

in the current configuration. From the vector crossproduct of the base vectors tangential to the convective coordinates $\xi$ and $\eta$ we obtain:

$$
\overline{\boldsymbol{n}}=\frac{\boldsymbol{x}_{, \xi} \times \boldsymbol{x}_{, \eta}}{\left|\boldsymbol{x}_{, \xi} \times \boldsymbol{x}_{, \eta}\right|}=\frac{\boldsymbol{n}}{|\boldsymbol{n}|} .
$$


In the further developments we only need the non-normalized normal vector $\boldsymbol{n}$ on $d \Gamma$, because the absolute values $|\boldsymbol{n}|$ in (2.16) and (2.17) cancel each other in the corresponding surface integrals e.g. in equations (2.11), (2.12) and (2.14).

It is obvious that the gas and the fluid volume are not only bounded by the structural

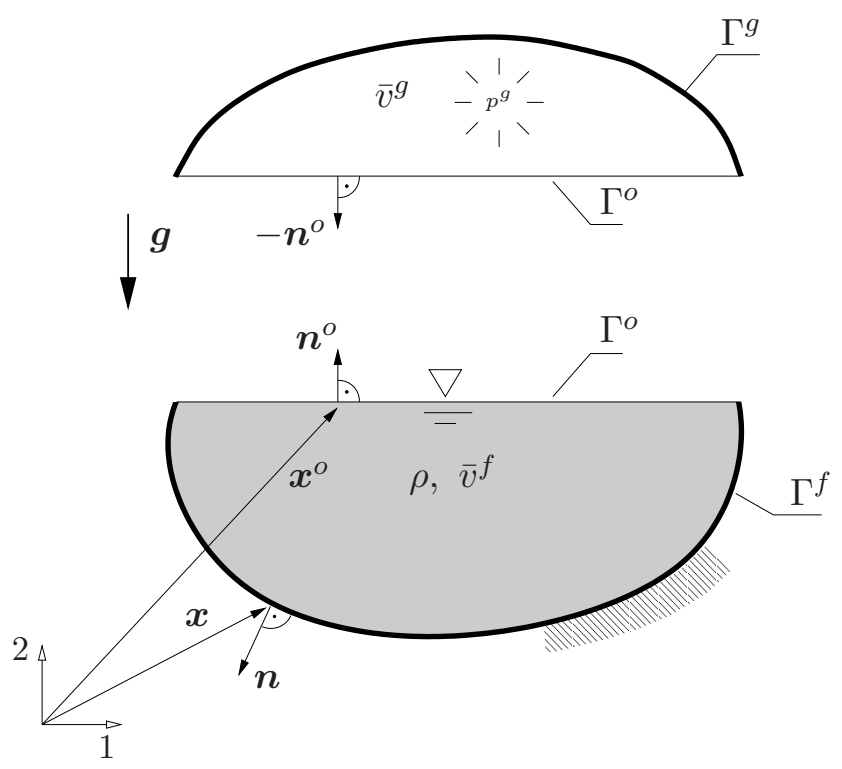

Fig. 2.3: Sectioning of control volumes $v^{g}$ and $v^{f}$

surface (leading to the projections $\delta v^{g}, \delta v^{f}$ and $\delta \boldsymbol{s}^{f}$ ) but also by the free fluid surface $\Gamma^{o}$. Therefore the terms $\delta \bar{v}^{g}, \delta \bar{v}^{f}$ and $\delta \overline{\boldsymbol{s}}$ in equations (2.11)-(2.14) also include the free fluid surface $\Gamma^{o}$ as a boundary, which leads to the projections $\delta v^{o}$ and $\delta \boldsymbol{s}^{o}$. To compute the volume change $\delta v^{o}$ under the fluid level we refer to equation (2.10), which equals $p^{k g}$ and $p^{k f}$ for the standard case of a single chamber. As both pressures are equal, their changes

$$
\delta p^{k g}=-\kappa \frac{p^{k g}}{\bar{v}^{g}} \delta \bar{v}^{g}=-\alpha \delta \bar{v}^{g}
$$

and

$$
\delta p^{k f}=-\frac{K}{\bar{v}_{0}^{f}} \delta \bar{v}^{f}=-\beta \delta \bar{v}^{f}
$$

also have to be identical.

$$
\begin{aligned}
\delta p^{k g} & =\delta p^{k f} \\
-\alpha \delta \bar{v}^{g} & =-\beta \delta \bar{v}^{f}
\end{aligned}
$$

Inserting (2.11) and (2.12) into equation (2.20) leads to the volume change $\delta v^{o}$.

$$
\begin{aligned}
-\alpha\left(\delta v^{g}-\delta v^{o}\right) & =-\beta\left(\delta v^{f}+\delta v^{o}\right) \\
\delta v^{o}(\alpha+\beta) & =\alpha \delta v^{g}-\beta \delta v^{f} \\
\delta v^{o} & =\frac{\alpha}{\alpha+\beta} \delta v^{g}-\frac{\beta}{\alpha+\beta} \delta v^{f}
\end{aligned}
$$


For reasons of convenience we introduce the dimensionless pressure volume gradients

$$
\bar{\alpha}=\frac{\alpha}{\alpha+\beta}
$$

and

$$
\bar{\beta}=\frac{\beta}{\alpha+\beta} .
$$

This leads to

$$
\delta v^{o}=\bar{\alpha} \delta v^{g}-\bar{\beta} \delta v^{f}
$$

for the volume change under the fluid level (see also figure 2.4). For the derivations following later the features of the stiffness parameters $\bar{\alpha}$ and $\bar{\beta}$ will be briefly outlined here.

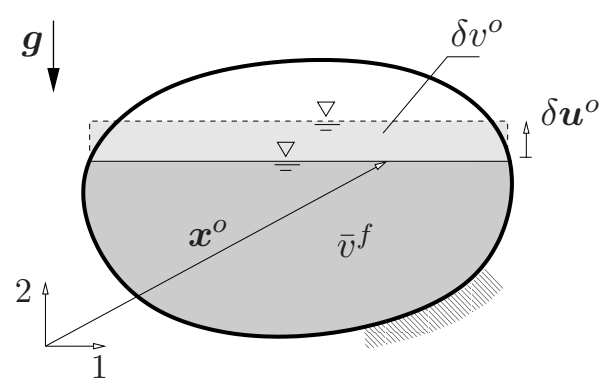

Fig. 2.4: First variation of volume change $\delta v^{o}$ under the fluid level $\Gamma^{o}$

\subsubsection{Only gas filling}

A chamber, as shown in figure 2.3, with a negligible initial fluid volume $\bar{v}_{0}^{f} \rightarrow 0$ results in an infinite pressure volume gradient

$$
\lim _{\bar{v}_{0}^{f} \rightarrow 0} \beta=\lim _{\bar{v}_{0}^{f} \rightarrow 0} \frac{K}{\bar{v}_{0}^{f}}=\infty
$$

This gives us

$$
\bar{\alpha}=0 \quad \text { and } \quad \bar{\beta}=1
$$

for a chamber only filled with gas.

\subsubsection{Complete fluid filling}

Considering a chamber with a negligible gas volume $\bar{v}^{g} \rightarrow 0$ and nearly completely filled with fluid the limit of the pressure volume gradient $\alpha$ - under the assumption that a finite gas pressure $p^{k g}$ is at hand - becomes

$$
\lim _{\bar{v}^{g} \rightarrow 0} \alpha=\lim _{\bar{v}^{g} \rightarrow 0} \kappa \frac{p^{k g}}{\bar{v}^{g}}=\infty .
$$

In this case of a chamber only filled with fluid we obtain

$$
\bar{\alpha}=1 \text { and } \bar{\beta}=0 .
$$

The parameters $\bar{\alpha}$ and $\bar{\beta}$ thus allow a universal description of all possible combinations of fluid and gas filling in a simple fashion. 


\subsection{Virtual Work of the Absolute Fluid and Gas Pressures}

After all geometrical and constitutive equations have been set up, the virtual potential (2.2) by using equations (2.11) - (2.15), (2.16) and (2.17) can be written as:

$$
\begin{aligned}
\delta \Pi^{f s i}= & -\delta \rho \boldsymbol{g} \cdot \overline{\boldsymbol{s}}^{f}-\rho \boldsymbol{g} \cdot \delta \overline{\boldsymbol{s}}^{f}-p^{k f} \delta \bar{v}^{f}-p^{k g} \delta \bar{v}^{g} \\
= & \frac{\rho}{\bar{v}^{f}} \boldsymbol{g} \cdot \overline{\boldsymbol{s}}^{f}\left(\delta v^{f}+\delta v^{o}\right)-\rho \boldsymbol{g} \cdot\left(\delta \boldsymbol{s}^{f}+\delta \boldsymbol{s}^{o}\right) \\
& -p^{k f}\left(\delta v^{f}+\delta v^{o}\right)-p^{k g}\left(\delta v^{g}-\delta v^{o}\right) \\
= & \frac{\rho}{\bar{v}^{f}} \boldsymbol{g} \cdot \overline{\boldsymbol{s}}^{f}\left(\delta v^{f}+\delta v^{o}\right)-\rho \boldsymbol{g} \cdot\left(\delta \boldsymbol{s}^{f}+\boldsymbol{x}^{o} \delta v^{o}\right) \\
& -p^{k f}\left(\delta v^{f}+\delta v^{o}\right)-p^{k g}\left(\delta v^{g}-\delta v^{o}\right) \\
= & \frac{\rho}{\bar{v}^{f}} \boldsymbol{g} \cdot \overline{\boldsymbol{s}}^{f}\left(\delta v^{f}+\bar{\alpha} \delta v^{g}-\bar{\beta} \delta v^{f}\right)-\rho \boldsymbol{g} \cdot\left(\delta \boldsymbol{s}^{f}+\boldsymbol{x}^{o}\left[\bar{\alpha} \delta v^{g}-\bar{\beta} \delta v^{f}\right]\right) \\
& -p^{k f}\left(\delta v^{f}+\bar{\alpha} \delta v^{g}-\bar{\beta} \delta v^{f}\right)-p^{k g}\left(\delta v^{g}-\bar{\alpha} \delta v^{g}+\bar{\beta} \delta v^{f}\right) .
\end{aligned}
$$

Dividing the first order volume moment $\bar{s}^{f}$ by the fluid volume $\bar{v}^{f}$ yields the center of gravity of the fluid.

$$
c^{f}=\frac{\bar{s}^{f}}{\bar{v}^{f}}
$$

Introducing the pressure in the center of gravity of the fluid

$$
p^{c}=\rho \boldsymbol{g} \cdot \boldsymbol{c}^{f}
$$

and the pressure at the fluid level

$$
p^{o}=\rho \boldsymbol{g} \cdot \boldsymbol{x}^{o}
$$

and rewriting (2.29) finally results in a generalized form of the variation of the fluid potential of a chamber partially filled with a compressible heavy fluid and gas, which is only described in terms of the surrounding shell surface $\Gamma^{g}$ and $\Gamma^{f}$.

$$
\begin{aligned}
\delta \Pi^{f s i}= & {\left[(1-\bar{\beta}) p^{c}-(1-\bar{\beta}) p^{k f}-\bar{\beta} p^{k g}+\bar{\beta} p^{o}\right] \delta v^{f} } \\
& +\left[\bar{\alpha}\left(p^{c}-p^{o}\right)-(1-\bar{\alpha}) p^{k g}-\bar{\alpha} p^{k f}\right] \delta v^{g}-\rho \boldsymbol{g} \cdot \delta \boldsymbol{s}^{f}
\end{aligned}
$$

Although equation (2.33) contains - due to $p^{k f}=p^{k g}$ — the vanishing terms

$$
\bar{\beta} p^{k f}-\bar{\beta} p^{k g}=0, \quad \text { resp. } \quad \bar{\alpha} p^{k g}-\bar{\alpha} p^{k f}=0,
$$

these terms will be kept in the formulations in order to derive some specific cases, which have already been discussed separately in [18], [19], [20] and [21].

\subsection{Derivation of Specific Cases}

Due to the fact that the gravitational pressures $p^{o}, p^{x}$ and $p^{c}$ result from a potential formulation they have to be multiplied by -1 to obtain a physical working pressure. For a better interpretation in the following figures, which will focus on some special load cases, these physical pressure terms are used. 


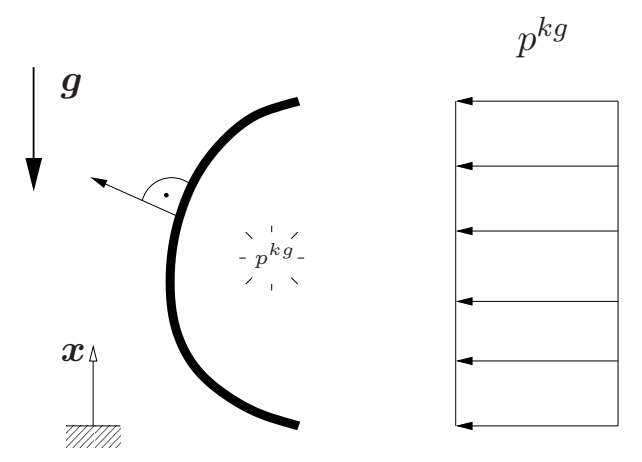

Fig. 2.5: Hydrostatic pressure distribution in a single chamber filled with gas

\subsubsection{Gas filled chamber}

In this case no fluid boundary $\Gamma^{f}$ and no fluid volume $v^{f}$ exists, therefore all terms defined by the fluid surface integrals and the fluid volume vanish. Further, no fluid level $\Gamma^{o}$ exists, which means no volume $v^{o}$ and therefore no volume change $\delta v^{o}$ can arise. Using $\delta v^{f}=0$ in equation (2.24) yields

$$
\bar{\alpha} \delta v^{g}=0 \text {. }
$$

This can only be satisfied if $\bar{\alpha}=0$ (because $\delta v^{g} \neq 0$ ), which follows directly from equation (2.26). For the variation of the $f s i$-potential $\delta \Pi^{f s i}$ in equation (2.33) only the virtual work of the gas compression remains (see also figure 2.5):

$$
\delta \Pi^{f s i}=-\delta W^{g}=-p^{k g} \delta v^{g}=-p^{k g} \int_{\eta^{g}} \int_{\xi^{g}} \boldsymbol{n}^{g} \cdot \delta \boldsymbol{u}^{g} d \xi d \eta
$$

\subsubsection{Incompressible fluid with free fluid surface}

In this case we consider containments with partial fluid filling, where e.g. a vent hole is existing in the non-wetted region ensuring that no overpressure can arise in the interior. Thus all gas pressure terms can be neglected. In the case of an incompressible fluid, which
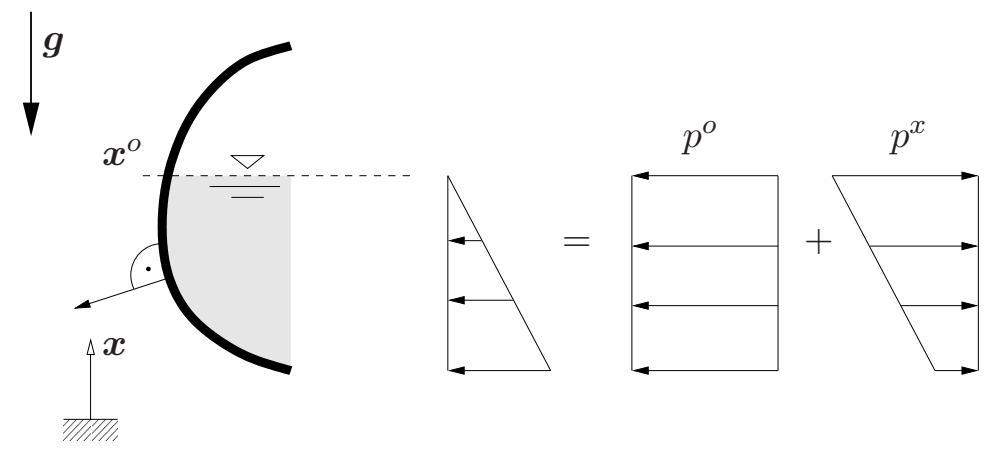

Fig. 2.6: Hydrostatic pressure distribution in a single chamber partially filled with an incompressible heavy fluid

means $K \rightarrow \infty$, the limit for the pressure volume gradient $\beta$ becomes:

$$
\lim _{K \rightarrow \infty} \beta=\lim _{K \rightarrow \infty} \frac{K}{\bar{v}^{f}}=\infty
$$


Thus for the dimensionless parameters $\bar{\alpha}$ and $\bar{\beta}$ the limits for $\beta \rightarrow \infty$ are

$$
\lim _{\beta \rightarrow \infty} \bar{\alpha}=\lim _{\beta \rightarrow \infty} \frac{\alpha}{\alpha+\beta}=0
$$

and

$$
\lim _{\beta \rightarrow \infty} \bar{\beta}=\lim _{\beta \rightarrow \infty} \frac{\beta}{\alpha+\beta}=\lim _{\beta \rightarrow \infty} \frac{1}{\frac{\alpha}{\beta}+1}=1 .
$$

Hence, for an incompressible fluid with a free fluid surface we obtain

$$
\bar{\alpha}=0 \quad \text { and } \quad \bar{\beta}=1 .
$$

Thus with (2.40) and $p^{k g}=0$ all gas terms are canceled in equation (2.33) and the variation of the potential becomes

$$
\begin{aligned}
\delta \Pi^{f s i} & =p^{o} \delta v^{f}-\rho \boldsymbol{g} \cdot \delta \boldsymbol{s}^{f} \\
& =\int_{\eta^{f}} \int_{\xi^{f}}\left(p^{o}-\rho \boldsymbol{g} \cdot \boldsymbol{x}\right) \boldsymbol{n}^{f} \cdot \delta \boldsymbol{u}^{f} d \xi d \eta \\
& =\int_{\eta^{f}} \int_{\xi^{f}}\left(p^{o}-p^{x}\right) \boldsymbol{n}^{f} \cdot \delta \boldsymbol{u}^{f} d \xi d \eta,
\end{aligned}
$$

with the gravitational pressure terms

$$
p^{x}=\rho \boldsymbol{g} \cdot \boldsymbol{x}
$$

denoting the pressure at a structural surface point on $\Gamma^{f}$ and

$$
p^{o}=\rho \boldsymbol{g} \cdot \boldsymbol{x}^{o}
$$

denoting the constant pressure at the fluid surface $\Gamma^{\circ}$ (see also figure 2.6) .

\subsubsection{Incompressible fluid with free fluid surface and additional gas loading}

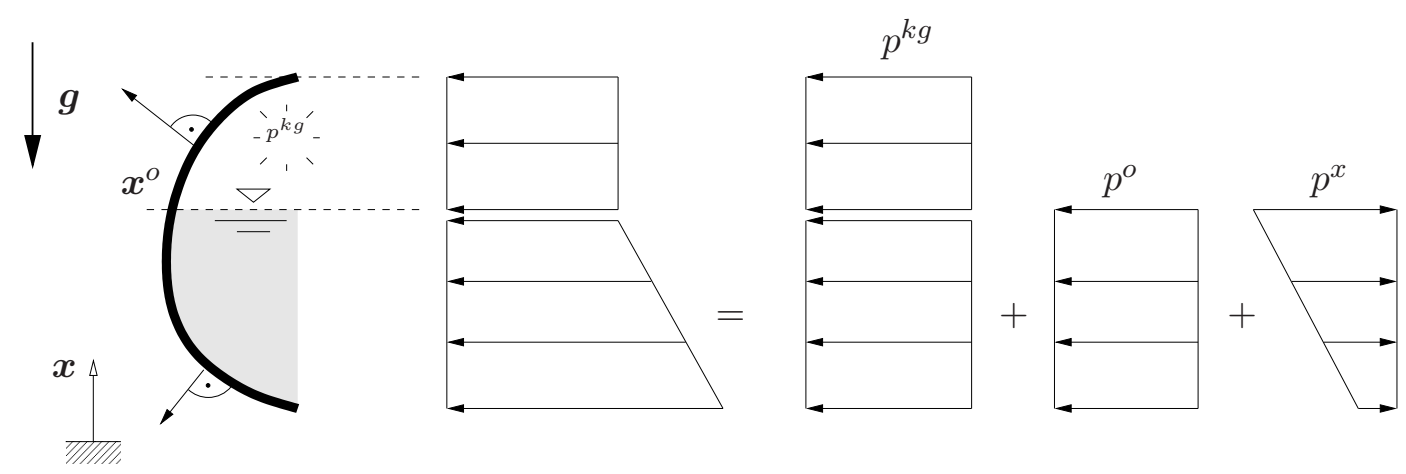

Fig. 2.7: Hydrostatic pressure distribution in a chamber filled with gas and an incompressible heavy fluid

For completely closed chambers an additional gas boundary $\Gamma^{g}$ appears. Therefore two gas terms remain in equation (2.33) resulting in the variation of the gas and the fluid potential

$$
\begin{aligned}
\delta \Pi^{f s i} & =\left(-p^{k g}+p^{o}\right) \delta v^{f}-\rho \boldsymbol{g} \cdot \delta \boldsymbol{s}^{f}-p^{k g} \delta v^{g} \\
& =\int_{\eta^{f}} \int_{\xi^{f}}\left(p^{o}-p^{x}-p^{k g}\right) \boldsymbol{n}^{f} \cdot \delta \boldsymbol{u}^{f} d \xi d \eta-\int_{\eta^{g}} \int_{\xi^{g}} p^{k g} \boldsymbol{n}^{g} \cdot \delta \boldsymbol{u}^{g} d \xi d \eta .
\end{aligned}
$$

The gas and fluid pressure distribution is depicted in figure 2.7. 


\subsubsection{Compressible fluid with free fluid surface}

As in section 2.5.2, all surface integrals over the gas domain can be removed, as no gas boundary $\Gamma^{g}$ exists. Therefore the gas pressure $p^{k g}$ is zero, which means $\alpha\left(p^{k g}\right)=0$. For the stiffness parameters $\bar{\alpha}$ and $\bar{\beta}$ then follows

$$
\bar{\alpha}=0, \quad \bar{\beta}=1
$$

As already mentioned in section 2.3 for a heavy fluid, which is only influenced by the gravity field, we consider no further volume compression. This is reflected by $\bar{\beta}=1$ canceling the term $p^{k f}$ in equation (2.33). Thus a compressible heavy fluid in absence of an exterior pressure is treated like an incompressible heavy fluid.

$$
\delta \Pi^{f s i}=\int_{\eta^{f}} \int_{\xi^{f}}\left(p^{o}-p^{x}\right) \boldsymbol{n}^{f} \cdot \delta \boldsymbol{u}^{f} d \xi d \eta
$$

\subsubsection{Chamber completely filled with a compressible fluid}
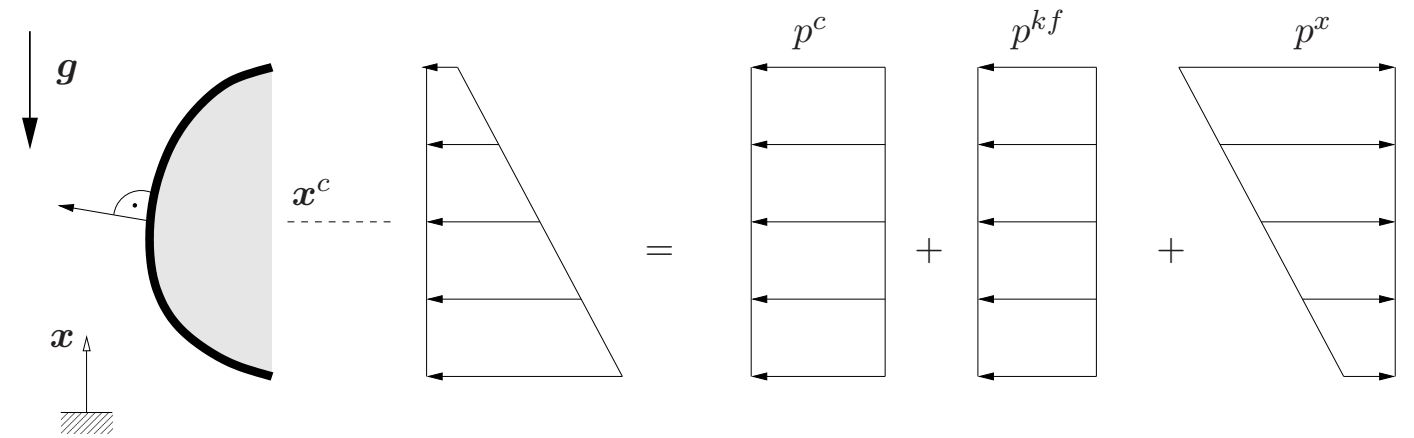

Fig. 2.8: Hydrostatic pressure distribution in a chamber completely filled with a compressible heavy fluid

For chambers completely filled with fluid the deformation of the surrounding structure in general goes along with both a volumetric and a deviatoric deformation. Therefore the compressibility of the fluid must be considered, because otherwise no volume change of the structure would be possible at all. Again no gas parts appear in the formulation for the variation of the potential and also no free fluid surface parts $\left(\Gamma^{o}=0\right)$, which is reflected in the stiffness parameters

$$
\bar{\alpha}=1 \text { and } \bar{\beta}=0,
$$

see also equation (2.28). Thus only the fluid potential terms remain with equation (2.42):

$$
\begin{aligned}
\delta \Pi^{f s i} & =\left(p^{c}-p^{k f}\right) \delta v^{f}-\rho \boldsymbol{g} \cdot \delta \boldsymbol{s}^{f} \\
& =\int_{\eta^{f}} \int_{\xi^{f}}\left(p^{c}-p^{k f}-p^{x}\right) \boldsymbol{n}^{f} \cdot \delta \boldsymbol{u}^{f} d \xi d \eta
\end{aligned}
$$

\subsubsection{Chamber containing compressible fluid and gas}

In the most general case all terms remain in the variation of the fluid and the gas potential. With $p^{k g}=p^{k f}$ and the introduction of the fluid pressure

$$
p^{f}=(1-\bar{\beta}) p^{c}-p^{k g}+\bar{\beta} p^{o}-p^{x}
$$




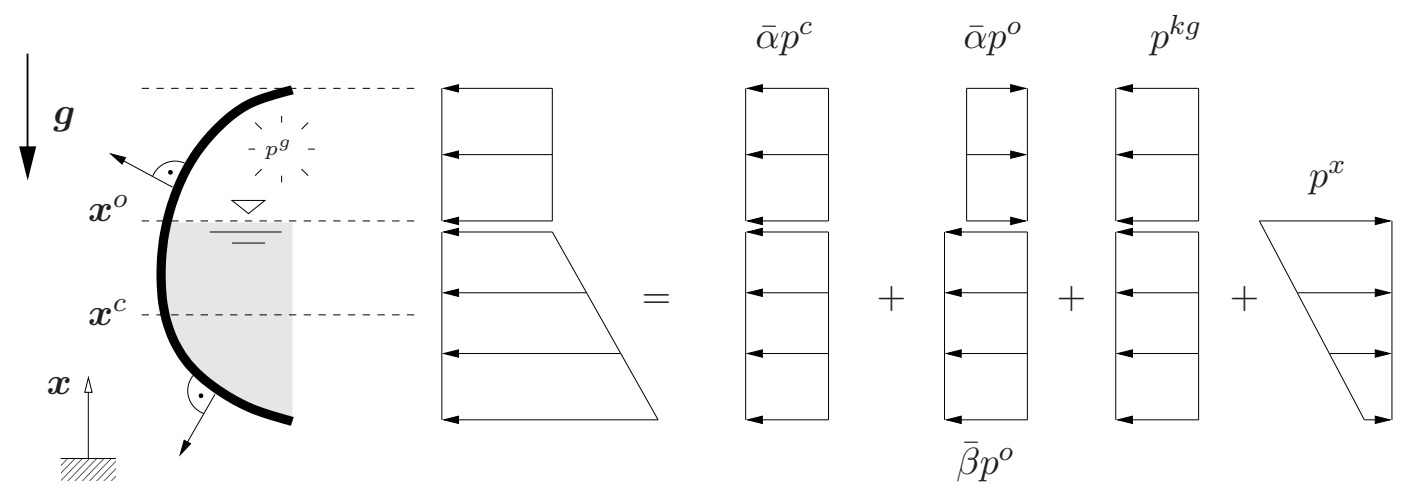

Fig. 2.9: Hydrostatic pressure distribution in a chamber filled with gas and a compressible heavy fluid

and the pressure inside the gas volume

$$
p^{g}=\bar{\alpha}\left(p^{c}-p^{o}\right)-p^{k g}
$$

from equation (2.33) the following variation of the potential is obtained

$$
\begin{aligned}
\delta \Pi^{f s i} & =\left[(1-\bar{\beta}) p^{c}-p^{k g}+\bar{\beta} p^{o}\right] \delta v^{f}-\rho \boldsymbol{g} \cdot \delta \boldsymbol{s}^{f}+\left[\bar{\alpha}\left(p^{c}-p^{o}\right)-p^{k g}\right] \delta v^{g} \\
& =\int_{\eta^{f}} \int_{\xi^{f}} p^{f} \boldsymbol{n}^{f} \cdot \delta \boldsymbol{u}^{f} d \xi d \eta+\int_{\eta^{g}} \int_{\xi^{g}} p^{g} \boldsymbol{n}^{g} \cdot \delta \boldsymbol{u}^{g} d \xi d \eta
\end{aligned}
$$

for a chamber with a compressible fluid and gas. The identity of the pressures at the interface is easily computed:

$$
\begin{aligned}
p^{f}\left(\boldsymbol{x}^{o}\right) & =(1-\bar{\beta}) p^{c}-p^{k g}+\bar{\beta} p^{o}-p^{o} \\
& =(1-\bar{\beta}) p^{c}-p^{k g}-(1-\bar{\beta}) p^{o} .
\end{aligned}
$$

As both fluid and gas share a common volume $\bar{v}=\bar{v}^{f}+\bar{v}^{g}$ we can write according to equations (2.22) and (2.23)

$$
\bar{\alpha}=\frac{\alpha}{\alpha+\beta}=\frac{\alpha+\beta}{\alpha+\beta}-\frac{\beta}{\alpha+\beta}=1-\bar{\beta} .
$$

Inserting (2.53) in (2.52) then gives the fluid pressure at the phase interface

$$
p^{f}\left(\boldsymbol{x}^{o}\right)=\bar{\alpha}\left(p^{c}-p^{o}\right)-p^{k g},
$$

which is identical to the gas pressure in equation (2.50).

\section{Linearization}

In this section the virtual expression (2.33) and its constraint equations (2.6), (2.8) and (2.9) will be expanded into a Taylor series up to the first order term in order to embed them into a numerical solution procedure, e.g. in a Newton-type scheme. A consequent linearization of the virtual work (2.33) at a given state $t$ then leads to

$$
\begin{aligned}
\delta \Pi_{l i n}^{f s i}=\quad & \text { Residual } \\
& \int_{\eta^{f}} \int_{\xi^{f}} p_{t}^{f} \boldsymbol{n}_{t}^{f} \cdot \delta \boldsymbol{u}^{f} d \xi d \eta+\int_{\eta^{g}} \int_{\xi^{g}} p_{t}^{g} \boldsymbol{n}_{t}^{g} \cdot \delta \boldsymbol{u}^{g} d \xi d \eta
\end{aligned}
$$


- Pressure change parts

$$
\begin{aligned}
+ & \int_{\eta^{f}} \int_{\xi^{f}} \Delta p^{f} \boldsymbol{n}_{t}^{f} \cdot \delta \boldsymbol{u}^{f} d \xi d \eta+\int_{\eta^{g}} \int_{\xi^{g}} \Delta p^{g} \boldsymbol{n}_{t}^{g} \cdot \delta \boldsymbol{u}^{g} d \xi d \eta \\
& \bullet \quad \text { Normal change parts } \\
+ & \int_{\eta^{f}} \int_{\xi^{f}} p_{t}^{f} \Delta \boldsymbol{n}^{f} \cdot \delta \boldsymbol{u}^{f} d \xi d \eta+\int_{\eta^{g}} \int_{\xi^{g}} p_{t}^{g} \Delta \boldsymbol{n}^{g} \cdot \delta \boldsymbol{u}^{g} d \xi d \eta .
\end{aligned}
$$

\subsection{Incremental pressure changes}

The pressure increments can be obtained by linearization of the fluid pressure

$$
\Delta p^{f}=(1-\bar{\beta}) \Delta p^{c}-(1-\bar{\beta}) \Delta p^{k f}-\bar{\beta} \Delta p^{k g}+\bar{\beta} \Delta p^{o}-\Delta p^{x}
$$

and the gas pressure

$$
\Delta p^{g}=\bar{\alpha}\left(\Delta p^{c}-\Delta p^{o}\right)-(1-\bar{\alpha}) \Delta p^{k g}-\bar{\alpha} \Delta p^{k f} .
$$

They can be expressed in terms of the incremental pressures $\Delta p^{c}, \Delta p^{o}, \Delta p^{k g}, \Delta p^{k f}$ and $\Delta p^{x}$, which can be obtained by linearization of the constitutive equations (2.8) and (2.9) along with the incremental changes of the geometrical quantities.

\subsubsection{Incremental changes of the geometrical quantities}

The identity of the pressures due to volume compression (2.10) allows to compute the volume change due to the height of the fluid level and the surface of the interface depending on the displacements of the wetted surfaces.

$$
\Delta v^{o}=\bar{\alpha} \Delta v^{g}-\bar{\beta} \Delta v^{f}
$$

Equations (2.11), (2.12) and (3.4) can then be used to derive the fluid volume change

$$
\begin{aligned}
\Delta \bar{v}^{f} & =\Delta\left(v^{f}+v^{o}\right) \\
& =\Delta v^{f}+\Delta v^{o} \\
& =\Delta v^{f}+\bar{\alpha} \Delta v^{g}-\bar{\beta} \Delta v^{f} \\
& =(1-\bar{\beta}) \Delta v^{f}+\bar{\alpha} \Delta v^{g}
\end{aligned}
$$

and the gas volume change

$$
\begin{aligned}
\Delta \bar{v}^{g} & =\Delta\left(v^{g}-v^{o}\right) \\
& =\Delta v^{g}-\Delta v^{o} \\
& =\Delta v^{g}-\left(\bar{\alpha} \Delta v^{g}-\bar{\beta} \Delta v^{f}\right) \\
& =(1-\bar{\alpha}) \Delta v^{g}+\bar{\beta} \Delta v^{f} .
\end{aligned}
$$

The incremental first order volume moment $\Delta \overline{\boldsymbol{s}}^{f}$ follows from equations (2.14) and (2.15) just by a change of the operator.

$$
\begin{aligned}
\Delta \bar{s}^{f} & =\Delta s^{f}+\Delta v^{o} \boldsymbol{x}_{t}^{o} \\
& =\Delta s^{f}+\left(\bar{\alpha} \Delta v^{g}-\bar{\beta} \Delta v^{f}\right) \boldsymbol{x}_{t}^{o}
\end{aligned}
$$

In analogy to (2.11) and (2.12) the incremental volume changes can be computed by a projection and subsequent surface integration of the structural displacements in normal direction.

$$
\Delta v^{f}=\int_{\eta^{f}} \int_{\xi^{f}} \boldsymbol{n}_{t}^{f} \cdot \Delta \boldsymbol{u}^{f} d \xi d \eta
$$




$$
\Delta v^{g}=\int_{\eta^{g}} \int_{\xi^{g}} \boldsymbol{n}_{t}^{g} \cdot \Delta \boldsymbol{u}^{g} d \xi d \eta
$$

In a similar fashion, see equation (2.14), the wetted surface part of the incremental first order volume moment is given as

$$
\Delta \boldsymbol{s}^{f}=\int_{\eta^{f}} \int_{\xi^{f}}\left(\boldsymbol{n}_{t}^{f} \cdot \Delta \boldsymbol{u}^{f}\right) \boldsymbol{x}_{t}^{f} d \xi d \eta .
$$

\subsubsection{Change of fluid level}

The incremental volume change of the fluid can also be seen as a change of the position $\boldsymbol{x}^{o}$ of the fluid surface $\Gamma_{t}^{o}$ by $\Delta \boldsymbol{u}^{o}$. Because $\Delta \boldsymbol{u}^{o}$ can only point into the direction of gravity, it can be moved out of the fluid surface integral by projecting $\Delta \boldsymbol{u}^{o}$ and $\boldsymbol{n}_{t}^{o}$ into the direction of $\boldsymbol{g}$. The remaining integral provides the total fluid surface $\Gamma_{t}^{o}$ at a given state $t$.

$$
\begin{aligned}
\Delta v^{o} & =\int_{\eta^{o}} \int_{\xi^{o}} \boldsymbol{n}_{t}^{o} \cdot \Delta \boldsymbol{u}^{o} d \xi d \eta \\
& =\int_{\eta^{o}} \int_{\xi^{o}} \boldsymbol{n}_{t}^{o} \cdot \frac{\boldsymbol{g}}{|\boldsymbol{g}|} \Delta \boldsymbol{u}^{o} \cdot \frac{\boldsymbol{g}}{|\boldsymbol{g}|} d \xi d \eta \\
& =\Delta \boldsymbol{u}^{o} \cdot \frac{\boldsymbol{g}}{|\boldsymbol{g}|} \int_{\eta^{o}} \int_{\xi^{o}} \boldsymbol{n}_{t}^{o} \cdot \frac{\boldsymbol{g}}{|\boldsymbol{g}|} d \xi d \eta \\
& =\Delta \boldsymbol{u}^{o} \cdot \frac{\boldsymbol{g}}{|\boldsymbol{g}|} \Gamma_{t}^{o}
\end{aligned}
$$

As the linearized state of equilibrium shall be written only in terms of the surrounding structure, we insert (3.4) into (3.11), which yields the correlation of $\Delta \boldsymbol{u}^{o}$ and the volume changes under the structure containing the gas and the fluid.

$$
\begin{aligned}
\Delta v^{o}=\Delta \boldsymbol{u}^{o} \cdot \frac{\boldsymbol{g}}{|\boldsymbol{g}|} \Gamma_{t}^{o} & =\bar{\alpha} \Delta v^{g}-\bar{\beta} \Delta v^{f} \\
\Delta \boldsymbol{u}^{o} \cdot \boldsymbol{g} & =\frac{|\boldsymbol{g}|}{\Gamma_{t}^{o}}\left(\bar{\alpha} \Delta v^{g}-\bar{\beta} \Delta v^{f}\right)
\end{aligned}
$$

\subsubsection{Pressure changes due to volume compression}

As already mentioned the pressure changes $\Delta p^{k f}$ and $\Delta p^{k g}$ are identical, but in order to keep a clear notation and a consequent derivation between all cases we continue using both pressure changes, which follow from equations (2.8), (2.9), (3.8) and (3.9).

$$
\begin{aligned}
\Delta p^{k f} & =-\beta_{t} \Delta \bar{v}^{f} \\
& =-\beta_{t}\left[(1-\bar{\beta}) \Delta v^{f}+\bar{\alpha} \Delta v^{g}\right] \\
& =-\beta_{t}(1-\bar{\beta}) \int_{\eta^{f}} \int_{\xi^{f}} \boldsymbol{n}_{t}^{f} \cdot \Delta \boldsymbol{u}^{f} d \xi d \eta-\beta_{t} \bar{\alpha} \int_{\eta^{g}} \int_{\xi^{g}} \boldsymbol{n}_{t}^{g} \cdot \Delta \boldsymbol{u}^{g} d \xi d \eta \\
\Delta p^{k g} & =-\alpha_{t} \Delta \bar{v}^{g} \\
& =-\alpha_{t}\left[(1-\bar{\alpha}) \Delta v^{g}+\bar{\beta} \Delta v^{f}\right] \\
& =-\alpha_{t}(1-\bar{\alpha}) \int_{\eta^{g}} \int_{\xi^{g}} \boldsymbol{n}_{t}^{g} \cdot \Delta \boldsymbol{u}^{g} d \xi d \eta-\alpha_{t} \bar{\beta} \int_{\eta^{f}} \int_{\xi^{f}} \boldsymbol{n}_{t}^{f} \cdot \Delta \boldsymbol{u}^{f} d \xi d \eta
\end{aligned}
$$




\subsubsection{Incremental pressure change in the center of gravity}

Linearization of equation (2.31) yields

$$
\begin{aligned}
\Delta p^{c} & =\Delta\left(\rho \boldsymbol{g} \cdot \frac{\overline{\boldsymbol{s}}}{\bar{v}^{f}}\right) \\
& =\Delta \rho \boldsymbol{g} \cdot \frac{\overline{\boldsymbol{s}}_{t}}{\bar{v}_{t}^{f}}+\rho_{t} \boldsymbol{g} \cdot \Delta\left(\frac{\bar{s}}{\bar{v}^{f}}\right) \\
& =\Delta \rho \boldsymbol{g} \cdot \boldsymbol{c}_{t}^{f}+\rho_{t} \boldsymbol{g} \cdot\left(\frac{\Delta \overline{\boldsymbol{s}}}{\bar{v}_{t}^{f}}-\Delta \bar{v}^{f} \frac{\overline{\boldsymbol{s}}_{t}}{\left(\bar{v}_{t}^{f}\right)^{2}}\right) .
\end{aligned}
$$

Due to mass conservation (2.6) and using equations (2.30), (2.31) along with (3.5) and (3.6) in equation (3.15) we obtain

$$
\begin{aligned}
\Delta p^{c}= & -\frac{\rho_{t}}{\bar{v}_{t}^{f}} \Delta \bar{v}^{f} \boldsymbol{g} \cdot \boldsymbol{c}_{t}+\rho_{t} \boldsymbol{g} \cdot\left(\frac{\Delta \overline{\boldsymbol{s}}}{\bar{v}_{t}^{f}}-\frac{\boldsymbol{c}_{t}^{f}}{\bar{v}_{t}^{f}} \Delta \bar{v}^{f}\right) \\
= & -2 \frac{p_{t}^{c}}{\bar{v}_{t}^{f}} \Delta \bar{v}^{f}+\frac{\rho_{t}}{\bar{v}_{t}^{f}} \boldsymbol{g} \cdot \Delta \overline{\boldsymbol{s}} \\
= & -2 \frac{p_{t}^{c}}{\bar{v}_{t}^{f}}\left[(1-\bar{\beta}) \Delta v^{f}+\bar{\alpha} \Delta v^{g}\right] \\
& +\frac{\rho_{t}}{\bar{v}_{t}^{f}} \boldsymbol{g} \cdot\left(\Delta \boldsymbol{s}^{f}+\bar{\alpha} \Delta v^{g} \boldsymbol{x}_{t}^{o}-\bar{\beta} \Delta v^{f} \boldsymbol{x}_{t}^{o}\right) .
\end{aligned}
$$

Rewriting equation (3.16) for the fluid and gas domains leads with

$$
p_{t}^{x}=\rho_{t} \boldsymbol{g} \cdot \boldsymbol{x}_{t}
$$

and equations (3.8) and (3.9) to the incremental pressure change $\Delta p^{c}$ in the center of gravity of the fluid as a function of the incremental displacements $\Delta \boldsymbol{u}^{g}$ and $\Delta \boldsymbol{u}^{f}$ of the surface wetted by the gas and the fluid:

$$
\begin{aligned}
\Delta p^{c}= & {\left[-2 \frac{p_{t}^{c}}{\bar{v}_{t}^{f}}(1-\bar{\beta})-\frac{p_{t}^{o}}{\bar{v}_{t}^{f}} \bar{\beta}\right] \Delta v^{f}+\left[\frac{p_{t}^{o}}{\bar{v}_{t}^{f}} \bar{\alpha}-2 \frac{p_{t}^{c}}{\bar{v}_{t}^{f}} \bar{\alpha}\right] \Delta v^{g}+\frac{\rho_{t}}{\bar{v}_{t}^{f}} \boldsymbol{g} \cdot \Delta \boldsymbol{s}^{f} } \\
= & \frac{1}{\bar{v}_{t}^{f}} \int_{\eta^{f}} \int_{\xi^{f}}\left[-2 p_{t}^{c}(1-\bar{\beta})-p_{t}^{o} \bar{\beta}+p_{t}^{x}\right] \boldsymbol{n}_{t}^{f} \cdot \Delta \boldsymbol{u}^{f} d \xi d \eta \\
& +\frac{1}{\bar{v}_{t}^{f}} \int_{\eta^{g}} \int_{\xi^{g}} \bar{\alpha}\left(p_{t}^{o}-2 p_{t}^{c}\right) \boldsymbol{n}_{t}^{g} \cdot \Delta \boldsymbol{u}^{g} d \xi d \eta
\end{aligned}
$$

\subsubsection{Incremental pressure change at the fluid level position}

Due to splitting up the fluid boundary into a part bounded by the structural surface $\Gamma^{f}$ and a part bounded by the fluid level $\Gamma^{o}$ the pressure $p^{o}$ at the fluid level had to be defined. Hence from the linearization of (2.32) follows with equations (2.6), (3.5) and (3.6)

$$
\begin{aligned}
\Delta p^{o} & =\Delta\left(\rho \boldsymbol{g} \cdot \boldsymbol{x}^{o}\right) \\
& =\Delta \rho \boldsymbol{g} \cdot \boldsymbol{x}_{t}^{o}+\rho_{t} \boldsymbol{g} \cdot \Delta \boldsymbol{u}^{o} \\
& =-\frac{\rho_{t}}{\bar{v}_{t}^{f}} \Delta \bar{v}^{f} \boldsymbol{g} \cdot \boldsymbol{x}_{t}^{o}+\rho_{t} \boldsymbol{g} \cdot \Delta \boldsymbol{u}^{o} \\
& =-\frac{\rho_{t}}{\bar{v}_{t}^{f}}\left[(1-\bar{\beta}) \Delta v^{f}+\bar{\alpha} \Delta v^{g}\right] \boldsymbol{g} \cdot \boldsymbol{x}_{t}^{o}+\rho_{t} \boldsymbol{g} \cdot \Delta \boldsymbol{u}^{o} .
\end{aligned}
$$


After inserting the projection of the displacement increment (3.12) we obtain

$$
\Delta p^{o}=-\frac{\rho_{t}}{\bar{v}_{t}^{f}}\left[(1-\bar{\beta}) \Delta v^{f}+\bar{\alpha} \Delta v^{g}\right] \boldsymbol{g} \cdot \boldsymbol{x}_{t}^{o}+\rho_{t} \frac{|\boldsymbol{g}|}{\Gamma_{t}^{o}}\left(\bar{\alpha} \Delta v^{g}-\bar{\beta} \Delta v^{f}\right) .
$$

Again the terms with the same integration domains can be collected and with the volume changes (3.8) and (3.9) and the pressure volume gradient

$$
\gamma_{t}=\frac{\rho_{t}|\boldsymbol{g}|}{\Gamma_{t}^{o}}
$$

the pressure change $\Delta p^{o}$ at $\boldsymbol{x}^{o}$ becomes

$$
\begin{aligned}
\Delta p^{o}= & {\left[-\frac{p_{t}^{o}}{\bar{v}_{t}^{f}}(1-\bar{\beta})-\frac{\rho_{t}|\boldsymbol{g}|}{\Gamma_{t}^{o}} \bar{\beta}\right] \Delta v^{f}+\bar{\alpha}\left(\frac{\rho_{t}|\boldsymbol{g}|}{\Gamma_{t}^{o}}-\frac{p_{t}^{o}}{\bar{v}_{t}^{f}}\right) \Delta v^{g} } \\
= & {\left[-\frac{p_{t}^{o}}{\bar{v}_{t}^{f}}(1-\bar{\beta})-\gamma_{t} \bar{\beta}\right] \Delta v^{f}+\bar{\alpha}\left(\gamma_{t}-\frac{p_{t}^{o}}{\bar{v}_{t}^{f}}\right) \Delta v^{g} } \\
= & {\left[-\frac{p_{t}^{o}}{\bar{v}_{t}^{f}}(1-\bar{\beta})-\gamma_{t} \bar{\beta}\right] \int_{\eta^{f}} \int_{\xi^{f}} \boldsymbol{n}_{t}^{f} \cdot \Delta \boldsymbol{u}^{f} d \xi d \eta } \\
& +\bar{\alpha}\left(\gamma_{t}-\frac{p_{t}^{o}}{\bar{v}_{t}^{f}}\right) \int_{\eta^{g}} \int_{\xi^{g}} \boldsymbol{n}_{t}^{g} \cdot \Delta \boldsymbol{u}^{g} d \xi d \eta
\end{aligned}
$$

\subsubsection{Incremental pressure change at a structural surface point wetted by the fluid}

As for $\Delta p^{o}$ in equation (3.22) we use the equations for the mass conservation (2.6) and for the volume changes (3.8) and (3.9) to derive the pressure change $\Delta p^{x}$ at a structural surface point $\boldsymbol{x}$ wetted by the fluid.

$$
\begin{aligned}
\Delta p^{x}= & \Delta(\rho \boldsymbol{g} \cdot \boldsymbol{x}) \\
= & \Delta \rho \boldsymbol{g} \cdot \boldsymbol{x}_{t}+\rho_{t} \boldsymbol{g} \cdot \Delta \boldsymbol{u} \\
= & -\frac{\rho_{t}}{\bar{v}_{t}^{f}} \Delta \bar{v}^{f} \boldsymbol{g} \cdot \boldsymbol{x}_{t}+\rho_{t} \boldsymbol{g} \cdot \Delta \boldsymbol{u} \\
= & -\frac{p_{t}^{x}}{\bar{v}_{t}^{f}}\left[(1-\bar{\beta}) \Delta v^{f}+\bar{\alpha} \Delta v^{g}\right]+\rho_{t} \boldsymbol{g} \cdot \Delta \boldsymbol{u} \\
= & -\frac{p_{t}^{x}}{\bar{v}_{t}^{f}}(1-\bar{\beta}) \int_{\eta^{f}} \int_{\xi^{f}} \boldsymbol{n}_{t}^{f} \cdot \Delta \boldsymbol{u}^{f} d \xi d \eta \\
& \quad-\frac{p_{t}^{x}}{\bar{v}_{t}^{f}} \bar{\alpha} \int_{\eta^{g}} \int_{\xi^{g}} \boldsymbol{n}_{t}^{g} \cdot \Delta \boldsymbol{u}^{g} d \xi d \eta+\rho_{t} \boldsymbol{g} \cdot \Delta \boldsymbol{u}
\end{aligned}
$$

\subsection{Terms including the change of the normals}

The terms describing the change of the normals for the wetted gas and fluid domains of the surrounding structure can be adapted from [19] and [20]. For the fluid domain the additional part due to the non-constant pressure distribution $p_{t}^{x}$ must be considered. By introducing the skewsymmetric tensors (see also [24] and [15])

$$
\begin{aligned}
\boldsymbol{W}^{\bar{\xi}} & =\boldsymbol{n}_{t} \otimes \boldsymbol{x}^{\bar{\xi}}-\boldsymbol{x}^{\bar{\xi}} \otimes \boldsymbol{n}_{t} \\
\boldsymbol{W}^{\bar{\eta}} & =\boldsymbol{n}_{t} \otimes \boldsymbol{x}^{, \bar{\eta}}-\boldsymbol{x}^{\bar{\eta}} \otimes \boldsymbol{n}_{t}
\end{aligned}
$$


in a convective basis $\boldsymbol{x}^{\bar{\xi}} \otimes \boldsymbol{x}^{, \bar{\eta}}$, with $\bar{\xi}, \bar{\eta}$ as the contravariant curvilinear coordinates, we obtain the normal change parts of the linearized virtual work as follows:

$$
\begin{aligned}
\delta \Pi_{l i n}^{\Delta n^{g}}= & \int_{\eta^{g}} \int_{\xi^{g}} \frac{p_{t}^{g}}{2}\left(\begin{array}{c}
\delta \boldsymbol{u}^{g} \\
\delta \boldsymbol{u}_{, \xi}^{g} \\
\delta \boldsymbol{u}_{, \eta}^{g}
\end{array}\right) \cdot\left(\begin{array}{ccc}
\mathbf{0} & \boldsymbol{W}^{\bar{\xi}} & \boldsymbol{W}^{\bar{\eta}} \\
\boldsymbol{W}^{\bar{\xi} T} & \mathbf{0} & \mathbf{0} \\
\boldsymbol{W}^{\bar{\eta} T} & \mathbf{0} & \mathbf{0}
\end{array}\right)\left(\begin{array}{c}
\Delta \boldsymbol{u}^{g} \\
\Delta \boldsymbol{u}_{, \xi}^{g} \\
\Delta \boldsymbol{u}_{, \eta}^{g}
\end{array}\right) d \xi d \eta \\
\delta \Pi_{l i n}^{\Delta n^{f}}= & \int_{\eta^{f}} \int_{\xi^{f}} \frac{p_{t}^{f}}{2}\left(\begin{array}{c}
\delta \boldsymbol{u}^{f} \\
\delta \boldsymbol{u}_{, \xi}^{f} \\
\delta \boldsymbol{u}_{, \eta}^{f}
\end{array}\right) \cdot\left(\begin{array}{ccc}
\mathbf{0} & \boldsymbol{W}^{\bar{\xi}} & \boldsymbol{W}^{\bar{\eta}} \\
\boldsymbol{W}^{\bar{\xi} T} & \mathbf{0} & \mathbf{0} \\
\boldsymbol{W}^{\bar{\eta} T} & \mathbf{0} & \mathbf{0}
\end{array}\right)\left(\begin{array}{c}
\Delta \boldsymbol{u}^{f} \\
\Delta \boldsymbol{u}_{, \xi}^{f} \\
\Delta \boldsymbol{u}_{, \eta}^{f}
\end{array}\right) d \xi d \eta \\
& -\int_{\eta^{f}} \int_{\xi^{f}}\left[p_{t, \xi}^{f}\left(\boldsymbol{x}_{, \eta} \times \delta \boldsymbol{u}_{, \xi}^{f}\right)-p_{t, \eta}^{f}\left(\boldsymbol{x}_{, \xi} \times \delta \boldsymbol{u}_{, \eta}^{f}\right)\right] \cdot \Delta \boldsymbol{u}^{f} d \xi d \eta .
\end{aligned}
$$

The position dependent pressure inside the fluid can be computed with

$$
p_{t}^{f}=(1-\bar{\beta}) p_{t}^{c}-(1-\bar{\beta}) p_{t}^{k f}-\bar{\beta} p_{t}^{k g}+\bar{\beta} p_{t}^{o}-p_{t}^{x},
$$

whereas the constant pressure inside the gas volume follows from

$$
p_{t}^{g}=\bar{\alpha}\left(p_{t}^{c}-p_{t}^{o}\right)-(1-\bar{\alpha}) p_{t}^{k g}-\bar{\alpha} p_{t}^{k f} .
$$

\subsection{Proof of Conservativeness}

As it was shown in [24] and [15] by product integration and subsequent application of the Gauss theorem the formally unsymmetric part in equation (3.27) can be transformed into a skew symmetric field term and an unsymmetric boundary term. Assuming physically realistic boundary conditions makes this boundary term vanish. A complete symmetry of the linearized virtual work $\delta \Pi_{\text {lin }}^{f s i}$ is finally obtained without any further assumption, if the linearized pressure parts (3.13), (3.14), (3.18), (3.22) and (3.23) along with the normal change parts (3.26) and (3.27) are inserted into the initial equation (3.1). If now the last term $\rho_{t} \boldsymbol{g} \cdot \Delta \boldsymbol{u}$ in equation (3.23) is split up into a symmetric and a skewsymmetric part, this skewsymmetric part neutralizes with the remaining skewsymmetric term in (3.27). Thus only the symmetric part from the position dependent pressure over the domain $\Gamma^{f}$ remains. The linearized virtual potential $\delta \Pi_{l i n}^{f s i}$ finally consists of the following five parts including the residual terms:

\section{Residual terms computed over $\Gamma^{f}$ and $\Gamma^{g}$}

$$
\begin{aligned}
\delta \Pi_{\text {lin }}^{r e s}= & \int_{\eta^{f}} \int_{\xi^{f}}\left[(1-\bar{\beta}) p_{t}^{c}-(1-\bar{\beta}) p_{t}^{k f}-\bar{\beta} p^{k g}+\bar{\beta} p_{t}^{o}-p_{t}^{x}\right] \boldsymbol{n}_{t}^{f} \cdot \delta \boldsymbol{u}^{f} d \xi d \eta \\
& +\int_{\eta^{g}} \int_{\xi^{g}}\left[\bar{\alpha}\left(p_{t}^{c}-p_{t}^{o}\right)-(1-\bar{\alpha}) p_{t}^{k g}-\bar{\alpha} p^{k f}\right] \boldsymbol{n}_{t}^{g} \cdot \delta \boldsymbol{u}^{g} d \xi d \eta
\end{aligned}
$$

\section{Coupling part computed over $\Gamma^{f}$}

$\delta \Pi_{\text {lin }}^{c p l, \Gamma^{f}}=$

- gravitational part in the center of gravity of the fluid: $(1-\bar{\beta}) \Delta p^{c} \delta v^{f}$

$$
\begin{aligned}
& \int_{\eta^{f}} \int_{\xi^{f}}\left[\frac{1-\bar{\beta}}{\bar{v}_{t}^{f}} \int_{\eta^{f}} \int_{\xi^{f}}\left[-2 p_{t}^{c}(1-\bar{\beta})-p_{t}^{o} \bar{\beta}+p_{t}^{x}\right] \boldsymbol{n}_{t}^{f} \cdot \Delta \boldsymbol{u}^{f} d \xi d \eta\right] \boldsymbol{n}_{t}^{f} \cdot \delta \boldsymbol{u}^{f} d \xi d \eta \\
& +\int_{\eta^{f}} \int_{\xi^{f}}\left[\frac{1-\bar{\beta}}{\bar{v}_{t}^{f}} \int_{\eta^{g}} \int_{\xi^{g}} \bar{\alpha}\left(p_{t}^{o}-2 p_{t}^{c}\right) \boldsymbol{n}_{t}^{g} \cdot \Delta \boldsymbol{u}^{g} d \xi d \eta\right] \boldsymbol{n}_{t}^{f} \cdot \delta \boldsymbol{u}^{f} d \xi d \eta
\end{aligned}
$$


- virtual work due to fluid volume compression: $-(1-\bar{\beta}) \Delta p^{k f} \delta v^{f}$

$$
\begin{aligned}
& -\int_{\eta^{f}} \int_{\xi^{f}}(1-\bar{\beta})\left[-\beta_{t}(1-\bar{\beta}) \int_{\eta^{f}} \int_{\xi^{f}} \boldsymbol{n}_{t}^{f} \cdot \Delta \boldsymbol{u}^{f} d \xi d \eta\right] \boldsymbol{n}_{t}^{f} \cdot \delta \boldsymbol{u}^{f} d \xi d \eta \\
& -\int_{\eta^{f}} \int_{\xi^{f}}(1-\bar{\beta})\left[-\beta_{t} \bar{\alpha} \int_{\eta^{g}} \int_{\xi^{g}} \boldsymbol{n}_{t}^{g} \cdot \Delta \boldsymbol{u}^{g} d \xi d \eta\right] \boldsymbol{n}_{t}^{f} \cdot \delta \boldsymbol{u}^{f} d \xi d \eta
\end{aligned}
$$

- virtual work due to gas volume compression: $-\bar{\beta} \Delta p^{k g} \delta v^{f}$

$$
\begin{aligned}
& -\int_{\eta^{f}} \int_{\xi^{f}} \bar{\beta}\left[-\alpha_{t}(1-\bar{\alpha}) \int_{\eta^{g}} \int_{\xi^{g}} \boldsymbol{n}_{t}^{g} \cdot \Delta \boldsymbol{u}^{g} d \xi d \eta\right] \boldsymbol{n}_{t}^{f} \cdot \delta \boldsymbol{u}^{f} d \xi d \eta \\
& -\int_{\eta^{f}} \int_{\xi^{f}} \bar{\beta}\left[-\alpha_{t} \bar{\beta} \int_{\eta^{f}} \int_{\xi^{f}} \boldsymbol{n}_{t}^{f} \cdot \Delta \boldsymbol{u}^{f} d \xi d \eta\right] \boldsymbol{n}_{t}^{f} \cdot \delta \boldsymbol{u}^{f} d \xi d \eta
\end{aligned}
$$

- gravitational part at the fluid level: $\bar{\beta} \Delta p^{o} \delta v^{f}$

$$
\begin{aligned}
& +\int_{\eta^{f}} \int_{\xi^{f}}\left[\bar{\beta}\left(-\frac{p_{t}^{o}}{\bar{v}_{t}^{f}}(1-\bar{\beta})-\gamma_{t} \bar{\beta}\right) \int_{\eta^{f}} \int_{\xi^{f}} \boldsymbol{n}_{t}^{f} \cdot \Delta \boldsymbol{u}^{f} d \xi d \eta\right] \boldsymbol{n}_{t}^{f} \cdot \delta \boldsymbol{u}^{f} d \xi d \eta \\
& +\int_{\eta^{f}} \int_{\xi^{f}}\left[\bar{\beta} \bar{\alpha}\left(\gamma_{t}-\frac{p_{t}^{o}}{\bar{v}_{t}^{f}}\right) \int_{\eta^{g}} \int_{\xi^{g}} \boldsymbol{n}_{t}^{g} \cdot \Delta \boldsymbol{u}^{g} d \xi d \eta\right] \boldsymbol{n}_{t}^{f} \cdot \delta \boldsymbol{u}^{f} d \xi d \eta
\end{aligned}
$$

- gravitational part: $-\Delta\left(\rho \boldsymbol{g} \cdot \delta \boldsymbol{s}^{f}\right)$ (without term $\left.\rho_{t} \boldsymbol{g} \cdot \Delta \boldsymbol{u}\right)$

$$
\begin{aligned}
& -\int_{\eta^{f}} \int_{\xi^{f}}\left[-\frac{p_{t}^{x}}{\bar{v}_{t}^{f}}(1-\bar{\beta}) \int_{\eta^{f}} \int_{\xi^{f}} \boldsymbol{n}_{t}^{f} \cdot \Delta \boldsymbol{u}^{f} d \xi d \eta\right] \boldsymbol{n}_{t}^{f} \cdot \delta \boldsymbol{u}^{f} d \xi d \eta \\
& -\int_{\eta^{f}} \int_{\xi^{f}}\left[-\frac{p_{t}^{x}}{\bar{v}_{t}^{f}} \bar{\alpha} \int_{\eta^{g}} \int_{\xi^{g}} \boldsymbol{n}_{t}^{g} \cdot \Delta \boldsymbol{u}^{g} d \xi d \eta\right] \boldsymbol{n}_{t}^{f} \cdot \delta \boldsymbol{u}^{f} d \xi d \eta
\end{aligned}
$$

\section{Coupling part computed over $\Gamma^{g}$}

$\delta \Pi_{l i n}^{c p l, \Gamma^{g}}=$

- gravitational part in the center of gravity of the fluid: $\bar{\alpha} \Delta p^{c} \delta v^{g}$

$$
\begin{aligned}
& \int_{\eta^{g}} \int_{\xi^{g}} \bar{\alpha}\left[\frac{1}{\bar{v}_{t}^{f}} \int_{\eta^{f}} \int_{\xi^{f}}\left[-2 p_{t}^{c}(1-\bar{\beta})-p_{t}^{o} \bar{\beta}+p_{t}^{x}\right] \boldsymbol{n}_{t}^{f} \cdot \Delta \boldsymbol{u}^{f} d \xi d \eta\right] \boldsymbol{n}_{t}^{g} \cdot \delta \boldsymbol{u}^{g} d \xi d \eta \\
& +\int_{\eta^{g}} \int_{\xi^{g}} \bar{\alpha}\left[\frac{1}{\bar{v}_{t}^{f}} \int_{\eta^{g}} \int_{\xi^{g}} \bar{\alpha}\left(p_{t}^{o}-2 p_{t}^{c}\right) \boldsymbol{n}_{t}^{g} \cdot \Delta \boldsymbol{u}^{g} d \xi d \eta\right] \boldsymbol{n}_{t}^{g} \cdot \delta \boldsymbol{u}^{g} d \xi d \eta
\end{aligned}
$$

- gravitational part: $-\bar{\alpha} \Delta p^{o} \delta v^{g}$

$$
\begin{aligned}
& -\int_{\eta^{g}} \int_{\xi^{g}} \bar{\alpha}\left[\left(-\frac{p_{t}^{o}}{\bar{v}_{t}^{f}}(1-\bar{\beta})-\gamma_{t} \bar{\beta}\right) \int_{\eta^{f}} \int_{\xi^{f}} \boldsymbol{n}_{t}^{f} \cdot \Delta \boldsymbol{u}^{f} d \xi d \eta\right] \boldsymbol{n}_{t}^{g} \cdot \delta \boldsymbol{u}^{g} d \xi d \eta \\
& -\int_{\eta^{g}} \int_{\xi^{g}} \bar{\alpha}\left[\bar{\alpha}\left(\gamma_{t}-\frac{p_{t}^{o}}{\bar{v}_{t}^{f}}\right) \int_{\eta^{g}} \int_{\xi^{g}} \boldsymbol{n}_{t}^{g} \cdot \Delta \boldsymbol{u}^{g} d \xi d \eta\right] \boldsymbol{n}_{t}^{g} \cdot \delta \boldsymbol{u}^{g} d \xi d \eta
\end{aligned}
$$

- virtual work due to gas volume compression: $-(1-\bar{\alpha}) \Delta p^{k g} \delta v^{g}$

$$
\begin{aligned}
& -\int_{\eta^{g}} \int_{\xi^{g}}(1-\bar{\alpha})\left[-\alpha_{t}(1-\bar{\alpha}) \int_{\eta^{g}} \int_{\xi^{g}} \boldsymbol{n}_{t}^{g} \cdot \Delta \boldsymbol{u}^{g} d \xi d \eta\right] \boldsymbol{n}_{t}^{g} \cdot \delta \boldsymbol{u}^{g} d \xi d \eta \\
& -\int_{\eta^{g}} \int_{\xi^{g}}(1-\bar{\alpha})\left[-\alpha_{t} \bar{\beta} \int_{\eta^{f}} \int_{\xi^{f}} \boldsymbol{n}_{t}^{f} \cdot \Delta \boldsymbol{u}^{f} d \xi d \eta\right] \boldsymbol{n}_{t}^{g} \cdot \delta \boldsymbol{u}^{g} d \xi d \eta
\end{aligned}
$$


- virtual work due to fluid volume compression: $-\bar{\alpha} \Delta p^{k f} \delta v^{g}$

$$
\begin{aligned}
& -\int_{\eta^{g}} \int_{\xi^{g}} \bar{\alpha}\left[-\beta_{t}(1-\bar{\beta}) \int_{\eta^{f}} \int_{\xi^{f}} \boldsymbol{n}_{t}^{f} \cdot \Delta \boldsymbol{u}^{f} d \xi d \eta\right] \boldsymbol{n}_{t}^{g} \cdot \delta \boldsymbol{u}^{g} d \xi d \eta \\
& -\int_{\eta^{g}} \int_{\xi^{g}} \bar{\alpha}\left[-\beta_{t} \bar{\alpha} \int_{\eta^{g}} \int_{\xi^{g}} \boldsymbol{n}_{t}^{g} \cdot \Delta \boldsymbol{u}^{g} d \xi d \eta\right] \boldsymbol{n}_{t}^{g} \cdot \delta \boldsymbol{u}^{g} d \xi d \eta
\end{aligned}
$$

Normal change part computed over $\Gamma^{g}$

$$
\delta \Pi_{l i n}^{\Delta n^{g}}=\frac{1}{2} \int_{\eta^{g}} \int_{\xi^{g}} p_{t}^{g}\left(\begin{array}{c}
\delta \boldsymbol{u}^{g} \\
\delta \boldsymbol{u}_{, \xi}^{g} \\
\delta \boldsymbol{u}_{, \eta}^{g}
\end{array}\right) \cdot\left(\begin{array}{ccc}
\mathbf{0} & \boldsymbol{W}^{\bar{\xi}} & \boldsymbol{W}^{\bar{\eta}} \\
\boldsymbol{W}^{\bar{\xi} T} & \mathbf{0} & \mathbf{0} \\
\boldsymbol{W}^{\bar{\eta} T} & \mathbf{0} & \mathbf{0}
\end{array}\right)\left(\begin{array}{c}
\Delta \boldsymbol{u}^{g} \\
\Delta \boldsymbol{u}_{, \xi}^{g} \\
\Delta \boldsymbol{u}_{, \eta}^{g}
\end{array}\right) d \xi d \eta
$$

Normal change part computed over $\Gamma^{f}$

For reasons of symmetry the term $\rho_{t} \boldsymbol{g} \cdot \Delta \boldsymbol{u}$ from the gravitational part $-\Delta\left(\rho \boldsymbol{g} \cdot \delta \boldsymbol{s}^{f}\right)$ was added:

$$
\begin{aligned}
\delta \Pi_{l i n}^{\Delta n^{f}}= & \frac{1}{2} \int_{\eta^{f}} \int_{\xi^{f}} p_{t}^{f}\left(\begin{array}{c}
\delta \boldsymbol{u}^{f} \\
\delta \boldsymbol{u}_{, \xi}^{f} \\
\delta \boldsymbol{u}_{, \eta}^{f}
\end{array}\right) \cdot\left(\begin{array}{ccc}
\mathbf{0} & \boldsymbol{W}^{\bar{\xi}} & \boldsymbol{W}^{\bar{\eta}} \\
\boldsymbol{W}^{\bar{\xi} T} & \mathbf{0} & \mathbf{0} \\
\boldsymbol{W}^{\bar{\eta} T} & \mathbf{0} & \mathbf{0}
\end{array}\right)\left(\begin{array}{c}
\Delta \boldsymbol{u}^{f} \\
\Delta \boldsymbol{u}_{, \xi}^{f} \\
\Delta \boldsymbol{u}_{, \eta}^{f}
\end{array}\right) d \xi d \eta \\
& -\frac{\rho_{t}}{2} \int_{\eta^{f}} \int_{\xi^{f}} \delta \boldsymbol{u}^{f} \cdot\left(\boldsymbol{n}_{t}^{f} \otimes \boldsymbol{g}+\boldsymbol{g} \otimes \boldsymbol{n}_{t}^{f}\right) \Delta \boldsymbol{u}^{f} d \xi d \eta .
\end{aligned}
$$

Thus for the linearized virtual fluid and gas potentials follows:

$$
\delta \Pi_{l i n}^{f s i}=\delta \Pi_{l i n}^{r e s}+\delta \Pi_{l i n}^{c p l, \Gamma^{f}}+\delta \Pi_{l i n}^{c p l, \Gamma^{g}}+\delta \Pi_{l i n}^{\Delta n^{g}}+\delta \Pi_{l i n}^{\Delta n^{f}} .
$$

The symmetry of this equation emerges even more clearly after discretization and a subsequent substitution of the integrals with vectors, which will be part of the next section.

\subsection{Finite Element Mapping}

After discretizing the linearized variation of the fluid structure interaction potential with isoparametric FE mapping functions $\boldsymbol{N}$ for the displacements, the virtual displacements and the incremental displacements of the structural parts which are in contact with gas/fluid, such that

$$
\boldsymbol{u}=\boldsymbol{N} \boldsymbol{d}, \quad \delta \boldsymbol{u}=\boldsymbol{N} \delta \boldsymbol{d} \quad \text { and } \quad \Delta \boldsymbol{u}=\boldsymbol{N} \Delta \boldsymbol{d}
$$

we obtain from the normal change parts of equation (3.33) and (3.34) the so-called load stiffness matrices for each structural element in contact with gas and/or fluid:

$$
\begin{aligned}
\boldsymbol{K}_{\text {elem }}^{g}= & \frac{1}{2} \int_{\eta^{g}} \int_{\xi^{g}} p_{t}^{g}\left(\begin{array}{c}
\boldsymbol{N}_{, \xi} \\
\boldsymbol{N}_{, \xi} \\
\boldsymbol{N}_{, \eta}
\end{array}\right)^{T}\left(\begin{array}{ccc}
\mathbf{0} & \boldsymbol{W}^{\bar{\xi}} & \boldsymbol{W}^{\bar{\eta}} \\
\boldsymbol{W}^{\xi \bar{T}} & \mathbf{0} & \mathbf{0} \\
\boldsymbol{W}^{\eta \bar{T}} & \mathbf{0} & \mathbf{0}
\end{array}\right)\left(\begin{array}{c}
\boldsymbol{N} \\
\boldsymbol{N}_{, \xi} \\
\boldsymbol{N}_{, \eta}
\end{array}\right) d \xi d \eta, \\
\boldsymbol{K}_{\text {elem }}^{f}= & \frac{1}{2} \int_{\eta^{f}} \int_{\xi^{f}} p_{t}^{f}\left(\begin{array}{c}
\boldsymbol{N}_{\boldsymbol{N}_{, \xi}} \\
\boldsymbol{N}_{, \eta}
\end{array}\right)^{T}\left(\begin{array}{ccc}
\mathbf{0} & \boldsymbol{W}^{\bar{\xi}} & \boldsymbol{W}^{\bar{\eta}} \\
\boldsymbol{W}^{\bar{\xi}} & \mathbf{0} & \mathbf{0} \\
\boldsymbol{W}^{\eta \bar{T}} & \mathbf{0} & \mathbf{0}
\end{array}\right)\left(\begin{array}{c}
\boldsymbol{N} \\
\boldsymbol{N}_{, \xi} \\
\boldsymbol{N}_{, \eta}
\end{array}\right) d \xi d \eta \\
& -\frac{\rho_{t}}{2} \int_{\eta^{f}} \int_{\xi^{f}} \boldsymbol{N}^{T}\left(\boldsymbol{n}_{t}^{f} \otimes \boldsymbol{g}+\boldsymbol{g} \otimes \boldsymbol{n}_{t}^{f}\right) \boldsymbol{N} d \xi d \eta
\end{aligned}
$$


whereas the residual for the corresponding element results in the negative right hand side vectors due to fluid and gas pressure loading

$$
\begin{array}{r}
\boldsymbol{f}_{\text {elem }}^{g}=\int_{\eta^{g}} \int_{\xi^{g}}\left[\bar{\alpha}\left(p_{t}^{c}-p_{t}^{o}\right)-(1-\bar{\alpha}) p_{t}^{k g}-\bar{\alpha} p_{t}^{k f}\right] \boldsymbol{N}^{T} \boldsymbol{n}_{t}^{g} d \xi d \eta, \\
\boldsymbol{f}_{\text {elem }}^{f}=\int_{\eta^{f}} \int_{\xi^{f}}\left[(1-\bar{\beta}) p_{t}^{c}-(1-\bar{\beta}) p_{t}^{k f}-\bar{\beta} p_{t}^{k g}\right. \\
\left.+\bar{\beta} p_{t}^{o}-p_{t}^{x}\right] \boldsymbol{N}^{T} \boldsymbol{n}_{t}^{f} d \xi d \eta .
\end{array}
$$

Hence along with the coupling vectors for the elements in contact with gas/fluid

$$
\begin{aligned}
& \boldsymbol{a}_{\text {elem }}=\int_{\eta^{g}} \int_{\xi^{g}} \boldsymbol{N}^{T} \boldsymbol{n}_{t}^{g} d \xi d \eta, \\
& \boldsymbol{b}_{\text {elem }}=\int_{\eta^{f}} \int_{\xi^{f}} \boldsymbol{N}^{T} \boldsymbol{n}_{t}^{f} d \xi d \eta \text { and } \\
& \boldsymbol{c}_{\text {elem }}=\int_{\eta^{f}} \int_{\xi^{f}} p_{t}^{x} \boldsymbol{N}^{T} \boldsymbol{n}_{t}^{f} d \xi d \eta
\end{aligned}
$$

as well as with the stiffness and residual parts $\boldsymbol{K}_{\text {elem }}^{\text {el }}$ and $\boldsymbol{f}_{\text {elem }}^{\text {el }}$ (deriving from the elastic shell structures) and the external load vector $\boldsymbol{f}^{e x}$ the arrays on element level can be computed. After assembling all local arrays in their corresponding global arrays we can formulate the linearized weak form of equilibrium of a fluid and gas filled structure (by taking into account the correct boundary conditions) as follows:

$$
\begin{aligned}
{[\boldsymbol{K}+} & \left.C_{1} \boldsymbol{b} \boldsymbol{b}^{T}+C_{2}\left(\boldsymbol{b} \boldsymbol{c}^{T}+\boldsymbol{c} \boldsymbol{b}^{T}\right)\right] \Delta \boldsymbol{d} \\
& +\left[C_{3} \boldsymbol{a} \boldsymbol{a}^{T}+C_{4}\left(\boldsymbol{a} \boldsymbol{c}^{T}+\boldsymbol{c} \boldsymbol{a}^{T}\right)+C_{5}\left(\boldsymbol{b} \boldsymbol{a}^{T}+\boldsymbol{a} \boldsymbol{b}^{T}\right)\right] \Delta \boldsymbol{d}=\boldsymbol{f},
\end{aligned}
$$

with the global system matrix

$$
\boldsymbol{K}=\boldsymbol{K}^{e l}+\boldsymbol{K}^{g}+\boldsymbol{K}^{f}
$$

and the right hand side vector

$$
\boldsymbol{f}=\boldsymbol{f}^{e x}-\boldsymbol{f}^{e l}-\boldsymbol{f}^{g}-\boldsymbol{f}^{f}
$$

with $\boldsymbol{f}^{e x}$ as external load vector and $\boldsymbol{f}^{e l}$ as the vector of the inner element forces - not including gas and fluid loading - and the pressure volume gradients

$$
\begin{aligned}
C_{1}= & \frac{1-\bar{\beta}}{\bar{v}_{t}^{f}}\left[-2 p_{t}^{c}(1-\bar{\beta})-p_{t}^{o} \bar{\beta}\right]+\beta_{t}(1-\bar{\beta})^{2}+\bar{\beta}^{2} \alpha_{t} \\
& +\bar{\beta}\left(-\frac{p_{t}^{o}}{\bar{v}_{t}^{f}}(1-\bar{\beta})-\gamma_{t} \bar{\beta}\right) \\
C_{2}= & \frac{1-\bar{\beta}}{\bar{v}_{t}^{f}} \\
C_{3}= & \frac{\bar{\alpha}^{2}}{\bar{v}_{t}^{f}}\left(p_{t}^{o}-2 p_{t}^{c}\right)-\bar{\alpha}^{2}\left(\gamma_{t}-\frac{p_{t}^{o}}{\bar{v}_{t}^{f}}\right)+\alpha_{t}(1-\bar{\alpha})^{2}+\bar{\alpha}^{2} \beta_{t} \\
C_{4}= & \frac{\bar{\alpha}}{\bar{v}_{t}^{f}} \\
C_{5}= & \frac{1-\bar{\beta}}{\bar{v}_{t}^{f}} \bar{\alpha}\left(p_{t}^{o}-2 p_{t}^{c}\right)+(1-\bar{\beta}) \beta_{t} \bar{\alpha}+\bar{\beta} \alpha_{t}(1-\bar{\alpha})+\bar{\beta} \bar{\alpha}\left(\gamma_{t}-\frac{p_{t}^{o}}{\bar{v}_{t}^{f}}\right) .
\end{aligned}
$$


Thus the structure of the incremental equations can be described such that the stiffness matrix has the form of the standard stiffness matrix of the structure which has contact to gas and fluid and/or has enclosed gas and/or fluid chambers. To the existing terms the socalled load stiffness matrix is added only for elements with surfaces wetted by gas or fluid. Further a number of dyadic updates is added depending on the type of filling or loading of the structure for each enclosed chamber. These updates lead to fully populated matrix parts for the unknowns associated to the wetted surfaces of the corresponding chambers. For the direct solution this is definitely leading to considerable additional computational effort. However, the special structure of dyadic updates can be used for an efficient solution scheme which is described later in section 7 .

\subsection{Hybrid Approach}

It is not mandatory to derive a totally displacement dependent weak form as it was done in (3.35) respectively in (3.44). An alternative can be found in a hybrid approach, for which the increments in equation (3.2) and (3.3) can be taken as additional hybrid unknowns. Hence with the coupling vectors (3.41), (3.42) and (3.43) the constraint equations (3.13), (3.14), (3.18), (3.22) and (3.23) for the hybrid unknowns can be rewritten:

$$
\begin{aligned}
& -\frac{1}{\alpha_{t}} \Delta p^{k g}-\hat{\boldsymbol{a}} \cdot \Delta \boldsymbol{d}=0, \quad \text { with } \quad \hat{\boldsymbol{a}}=((1-\bar{\alpha}) \boldsymbol{a}+\bar{\beta} \boldsymbol{b}) \\
& -\frac{1}{\beta_{t}} \Delta p^{k f}-\hat{\boldsymbol{b}} \cdot \Delta \boldsymbol{d}=0, \quad \text { with } \quad \hat{\boldsymbol{b}}=((1-\bar{\beta}) \boldsymbol{b}+\bar{\alpha} \boldsymbol{a}) \\
& \frac{1}{\gamma_{t}} \Delta p^{o}-\frac{p_{t}^{o}}{\gamma_{t}} \frac{\Delta \rho}{\rho_{t}}-(\bar{\alpha} \boldsymbol{a}-\bar{\beta} \boldsymbol{b}) \cdot \Delta \boldsymbol{d}=0, \\
& \bar{v}_{t}^{f} \Delta p^{c}-2 p_{t}^{c} \bar{v}_{t}^{f} \frac{\Delta \rho}{\rho_{t}}-p_{t}^{o}(\bar{\alpha} \boldsymbol{a}-\bar{\beta} \boldsymbol{b}) \cdot \Delta \boldsymbol{d}-\boldsymbol{c} \cdot \Delta \boldsymbol{d}=0 .
\end{aligned}
$$

Substituting (3.54) in (3.55) leads to

$$
\begin{aligned}
& \bar{v}_{t}^{f} \Delta p^{c}+\left(-2 p_{t}^{c} \bar{v}_{t}^{f}+\frac{p_{t}^{o 2}}{\gamma_{t}}\right) \frac{\Delta \rho}{\rho_{t}}-\frac{p_{t}^{o}}{\gamma_{t}} \Delta p^{o}-\boldsymbol{c} \cdot \Delta \boldsymbol{d}=0, \\
& \Delta p^{x}=p_{t}^{x} \frac{\Delta \rho}{\rho_{t}}+\rho_{t} \boldsymbol{g} \cdot \boldsymbol{N} \Delta \boldsymbol{d}, \\
& \frac{\Delta \rho}{\rho_{t}}=-\frac{1}{\bar{v}^{f}} \hat{\boldsymbol{b}} \cdot \Delta \boldsymbol{d} .
\end{aligned}
$$

With the constraint equations (3.52) - (3.58) and the global arrays (3.45) and (3.46) the linearized state of equilibrium can be written in a hybrid formulation as

$$
\left[\begin{array}{cccccc}
\boldsymbol{K} & \hat{\boldsymbol{b}} & -\hat{\boldsymbol{b}} & -\hat{\boldsymbol{a}} & \bar{\beta} \boldsymbol{b}-\bar{\alpha} \boldsymbol{a} & -\boldsymbol{c} \\
\hat{\boldsymbol{b}}^{T} & 0 & 0 & 0 & 0 & \bar{v}_{t}^{f} \\
-\hat{\boldsymbol{b}}^{T} & 0 & -\frac{1}{\beta_{t}} & 0 & 0 & 0 \\
-\hat{\boldsymbol{a}}^{T} & 0 & 0 & -\frac{1}{\alpha_{t}} & 0 & 0 \\
\bar{\beta} \boldsymbol{b}^{T}-\bar{\alpha} \boldsymbol{a}^{T} & 0 & 0 & 0 & \frac{1}{\gamma_{t}} & -\frac{p_{t}^{o}}{\gamma_{t}} \\
-\boldsymbol{c}^{T} & \bar{v}_{t}^{f} & 0 & 0 & -\frac{p_{t}^{o}}{\gamma_{t}} & -2 p_{t}^{c} \bar{v}_{t}^{f}+\frac{p_{t}^{o 2}}{\gamma_{t}}
\end{array}\right]\left[\begin{array}{c}
\Delta \boldsymbol{d} \\
\Delta p^{c} \\
\Delta p^{k f} \\
\Delta p^{k g} \\
\Delta p^{o} \\
\frac{\Delta \rho}{\rho_{t}}
\end{array}\right]=\left[\begin{array}{c}
\boldsymbol{f} \\
0 \\
0 \\
0 \\
0 \\
0
\end{array}\right]
$$

This form gives a particular insight into the structure of the global matrix and allows also a direct development of a pure displacement form. 


\subsection{Derivation of Special Load Cases}

As some specific load cases, which are included in this overall description, have already been described in [5], [18], [19] and [20] they are only briefly mentioned in tabular form, see table 3.1.

Table 3.1: Derivation of specific load cases by adjustment of stiffness parameters $\bar{\alpha}$ and $\bar{\beta}$

\begin{tabular}{|l|c|c|}
\hline load case & zero-arrays & stiffn. param. \\
\hline \hline pure gas filling & $\boldsymbol{K}^{f}=\mathbf{0}, \boldsymbol{b}=\boldsymbol{c}=\boldsymbol{f}^{f}=\mathbf{0}$ & $\bar{\alpha}=0, \bar{\beta}=1$ \\
\hline $\begin{array}{l}\text { incompressible heavy fluid } \\
\text { with free fluid surface }\end{array}$ & $\boldsymbol{K}^{g}=\mathbf{0}, \boldsymbol{a}=\boldsymbol{c}=\boldsymbol{f}^{g}=\mathbf{0}$ & $\bar{\alpha}=0, \bar{\beta}=1$ \\
\hline $\begin{array}{l}\text { incompressible heavy fluid } \\
\text { with gas loaded fluid surface }\end{array}$ & $\boldsymbol{c}=\mathbf{0}$ & $\bar{\alpha}=0, \bar{\beta}=1$ \\
\hline $\begin{array}{l}\text { compressible heavy fluid } \\
\text { without free fluid surface }\end{array}$ & $\boldsymbol{K}^{g}=\mathbf{0}, \boldsymbol{a}=\boldsymbol{f}^{g}=\mathbf{0}$ & $\bar{\alpha}=1, \bar{\beta}=0$ \\
\hline
\end{tabular}

\subsection{Compressible heavy fluid with free fluid surface and additional gas loading}

In this most general case all terms remain in equation (3.44). Because gas and fluid share a common volume the relation

$$
\bar{\beta}=1-\bar{\alpha}
$$

can be used to simplify the constants (3.47) - (3.51). In a next step from equations (3.44) the vectors with identical coefficients can be collected. Introducing the abbreviations

$$
\begin{gathered}
\bar{C}_{1}=-\bar{\alpha} \alpha_{t}+\frac{1}{2} \bar{\alpha}^{2}\left(\alpha_{t}+\beta_{t}\right)+\bar{\alpha}^{2} \frac{p_{t}^{o}}{\bar{v}_{t}^{f}}+\frac{1}{2} \alpha_{t}-\frac{1}{2} \bar{\alpha}^{2} \gamma_{t}-\bar{\alpha}^{2} \frac{p_{t}^{c}}{\bar{v}_{t}^{f}} \\
\bar{C}_{2}=\frac{\bar{\alpha}}{\bar{v}_{t}^{f}}, \quad \bar{C}_{3}=-\bar{\alpha} \frac{p_{t}^{o}}{\bar{v}_{t}^{f}}+\gamma_{t}\left(\bar{\alpha}-\frac{1}{2}\right) \quad \text { and } \quad \bar{C}_{4}=\bar{\alpha}\left(\gamma_{t}-\frac{p_{t}^{o}}{\bar{v}_{t}^{f}}\right)
\end{gathered}
$$

we obtain for the volume coupling part

$$
\begin{aligned}
\boldsymbol{K}^{\text {coupl }}= & 2 \bar{C}_{1}(\boldsymbol{a}+\boldsymbol{b})(\boldsymbol{a}+\boldsymbol{b})^{T}+\bar{C}_{2}\left[(\boldsymbol{a}+\boldsymbol{b}) \boldsymbol{c}^{T}+\boldsymbol{c}(\boldsymbol{a}+\boldsymbol{b})^{T}\right] \\
& +2 \bar{C}_{3} \boldsymbol{b} \boldsymbol{b}^{T}+\bar{C}_{4}\left(\boldsymbol{a} \boldsymbol{b}^{T}+\boldsymbol{b} \boldsymbol{a}^{T}\right) .
\end{aligned}
$$

By using the modified coupling vectors

$$
\hat{\boldsymbol{a}}=\bar{C}_{4} \boldsymbol{a}+\bar{C}_{3} \boldsymbol{b}, \quad \hat{\boldsymbol{b}}=\boldsymbol{a}+\boldsymbol{b} \quad \text { and } \quad \hat{\boldsymbol{c}}=\left[\bar{C}_{1}(\boldsymbol{a}+\boldsymbol{b})+\bar{C}_{2} \boldsymbol{c}\right]
$$


the coupling part $\boldsymbol{K}^{\text {coupl }}$ can be further reduced to a rank-4-update

$$
\boldsymbol{K}^{\text {coupl }}=\hat{\boldsymbol{a}} \boldsymbol{b}^{T}+\boldsymbol{b} \hat{\boldsymbol{a}}^{T}+\hat{\boldsymbol{c}} \hat{\boldsymbol{b}}^{T}+\hat{\boldsymbol{b}} \hat{\boldsymbol{c}}^{T} .
$$

Now the linearized set of equations for a compressible heavy fluid with a free fluid surface and additional gas loading can be written as:

$$
\begin{aligned}
\left(\boldsymbol{K}^{e l}+\boldsymbol{K}^{f}+\boldsymbol{K}^{g}\right. & \\
& \left.+\hat{\boldsymbol{a}} \boldsymbol{b}^{T}+\boldsymbol{b} \hat{\boldsymbol{a}}^{T}+\hat{\boldsymbol{c}} \hat{\boldsymbol{b}}^{T}+\hat{\boldsymbol{b}} \hat{\boldsymbol{c}}^{T}\right) \Delta \boldsymbol{d}=\boldsymbol{f}^{e x}-\boldsymbol{f}^{e l}-\boldsymbol{f}^{f}-\boldsymbol{f}^{g} .
\end{aligned}
$$

The residual vectors $\boldsymbol{f}^{g}$ and $\boldsymbol{f}^{f}$ can be simplified by using $p^{k g}=p^{k f}$ and condition (3.60).

$$
\begin{aligned}
& \boldsymbol{f}^{g}=\int_{\eta^{g}} \int_{\xi^{g}}\left(\bar{\alpha}\left(p_{t}^{c}-p_{t}^{o}\right)-p_{t}^{k g}\right) \boldsymbol{N}^{T} \boldsymbol{n}_{t}^{g} d \xi d \eta \\
& \boldsymbol{f}^{f}=\int_{\eta^{f}} \int_{\xi^{f}}\left(\bar{\alpha} p_{t}^{c}+(1-\bar{\alpha}) p_{t}^{o}-p_{t}^{x}-p_{t}^{k g}\right) \boldsymbol{N}^{T} \boldsymbol{n}_{t}^{f} d \xi d \eta
\end{aligned}
$$

The linearized set of equations can also be given in a hybrid form as:

$$
\left[\begin{array}{ccccc}
\boldsymbol{K} & \bar{\alpha} \hat{\boldsymbol{a}} & (\bar{\alpha}-1) \hat{\boldsymbol{a}} & \boldsymbol{b}-\bar{\alpha} \hat{\boldsymbol{a}} & -\boldsymbol{c} \\
\bar{\alpha} \hat{\boldsymbol{a}}^{T} & 0 & 0 & 0 & \bar{v}_{t}^{f} \\
(\bar{\alpha}-1) \hat{\boldsymbol{a}}^{T} & 0 & -\frac{1}{\alpha_{t}} & 0 & 0 \\
\boldsymbol{b}-\bar{\alpha} \hat{\boldsymbol{a}}^{T} & 0 & 0 & \frac{1}{\gamma_{t}} & -\frac{p_{t}^{o}}{\gamma_{t}} \\
-\boldsymbol{c}^{T} & \bar{v}_{t}^{f} & 0 & -\frac{p_{t}^{o}}{\gamma_{t}} & -2 p_{t}^{c} \bar{v}_{t}^{f}+\frac{p_{t}^{o}}{\gamma_{t}}
\end{array}\right]\left[\begin{array}{c}
\Delta \boldsymbol{d} \\
\Delta p^{c} \\
\Delta p^{k g} \\
\Delta p^{o} \\
\frac{\Delta \rho}{\rho_{t}}
\end{array}\right]=\left[\begin{array}{c}
\boldsymbol{f} \\
0 \\
0 \\
0 \\
0
\end{array}\right]
$$

\subsubsection{Multi chamber problems}

The derived procedure can directly be expanded to problems, where multiple fluid and/or gas filled chambers are connected to each other (see figure 3.1). For such systems the corresponding global arrays (load-stiffness matrices, coupling vectors and right hand side vectors denoted by index $i$ ) have to be set up for each chamber $i$ and must be summed up for all $n$ chambers.

$$
\begin{aligned}
& \boldsymbol{K \Delta} \boldsymbol{d}+\sum_{i=1}^{n}\left[\boldsymbol{K}_{i}^{g}+\boldsymbol{K}_{i}^{f}\right. \\
& \left.\quad+\hat{\boldsymbol{a}}_{i} \boldsymbol{b}_{i}^{T}+\boldsymbol{b}_{i} \hat{\boldsymbol{a}}_{i}^{T}+\hat{\boldsymbol{c}}_{i} \hat{\boldsymbol{b}}_{i}^{T}+\hat{\boldsymbol{b}}_{i} \hat{\boldsymbol{c}}_{i}^{T}\right] \Delta \boldsymbol{d}=\boldsymbol{f}^{e x}-\boldsymbol{f}^{e l}-\sum_{i=1}^{n}\left(\boldsymbol{f}_{i}^{g}+f_{i}^{f}\right)
\end{aligned}
$$

In the hybrid approach the number of unknowns increases for each chamber by the number of internal state variables, in addition to the expansion of the system matrix by adding the load-stiffness matrices and the matrices due to the coupling vectors as well as modifying the right hand side vector by the appropriate pressure parts.

\section{General Solution Scheme}

One possibility to solve the derived coupled problems is based on the direct use of the hybrid form (3.59). For direct solvers the typical sparse storage scheme for symmetric matrices featuring mostly zero entries is taken, which ensures that the storage capacity 


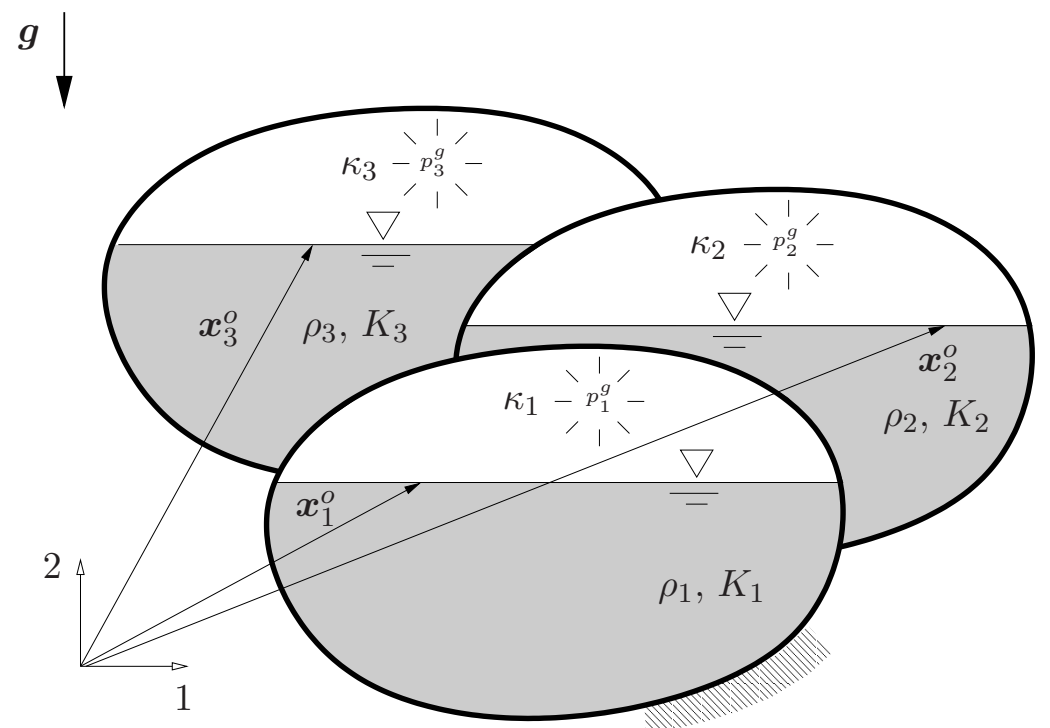

Fig. 3.1: Multichamber problem with 3 interacting fluid and gas filled chambers

is kept at a minimum and that the efficiency of a direct solver is not affected remarkably (assumed that the additional hybrid unknowns are only few compared to the original number of unknowns). Further on additional fill-in reducing strategies as nested dissection permutation [11] or minimum degree permutation [12] still guarantee an efficient $\boldsymbol{L}^{T} \boldsymbol{D} \boldsymbol{L}$ dissection of the system matrix. Nevertheless the fully populated rows and columns of the hybrid system matrix caused by the volume coupling lead to higher computational effort for its triangular factorization. Therefore in this section an alternative way will be presented, focusing on the solution of the fully displacement dependent formulation, which will arise if the additional unknowns have been eliminated and thus will lead to a dyadic updated system matrix. An additional advantage of the form with the dyadic rank updates can be seen in stability analyses, where the effect of each dyadic update on eigenvalues can be directly computed, see [13].

The derivation of the algorithm is described more detailed in appendix 7 at the example of a system matrix with a rank- $(m=3)$-update. According to this example a general algorithm for a system matrix $\boldsymbol{K}$ with $i=1 . . m$ dyadic rank updates $\boldsymbol{x}_{i} \boldsymbol{y}_{i}^{T}$

$$
\boldsymbol{A}=\boldsymbol{K}+\sum_{i=1}^{m} \boldsymbol{x}_{i} \boldsymbol{y}_{i}^{T}
$$

can be developed, which is depicted in figure 4.1 .

\subsection{Memory Requirements}

For each new recursive step the number of auxiliary vectors, which have to be stored, increases by one. Thus for the most general case

$$
\left(\boldsymbol{K}+\sum_{l=1}^{m} \boldsymbol{x}_{l} \boldsymbol{y}_{l}^{T}\right) \boldsymbol{d}=\boldsymbol{f}
$$




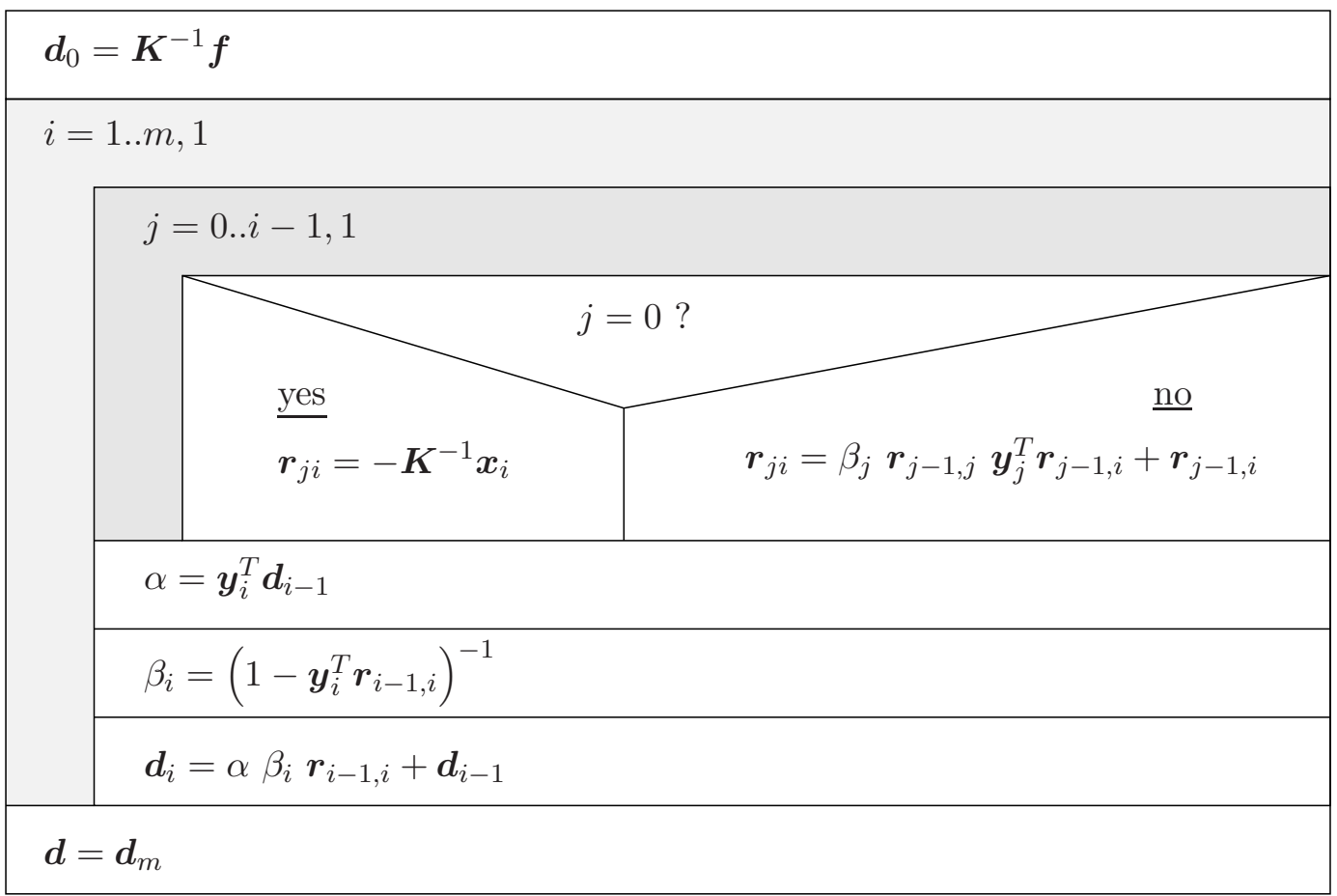

Fig. 4.1: Recursive scheme for the computation of the auxiliary vectors $\boldsymbol{r}_{j i}$, the parameters $\beta_{i}$ and the interim solutions $\boldsymbol{d}_{i}$ in the vectorized Sherman-Morrison algorithm for $m$ updates

of a rank- $m$-updated system matrix $\boldsymbol{K}$ the memory requirements listed also in table 4.1 are as follows:

- $k=\sum_{i=0}^{m}(m-i)$ auxiliary vectors $\boldsymbol{r}_{j i}$,

- $m$ parameters $\beta_{i}$,

- 2 interim solution vectors $\boldsymbol{d}_{i}$ and $\boldsymbol{d}_{i-1}$.

\subsection{Performance}

Although the hybrid system matrix from equation (3.59) is a sparse matrix, the $m$ fully populated rows and columns due to volume coupling lead to a higher computational effort for its triangular factorization than for the standard system matrix having a typical band structure. Therefore in the next step a comparison of the computational effort between the solution of the hybrid equation system and the fully displacement dependent equation system with the presented recursive algorithm will be performed. The operation counts for factorizations and forward-backward substitutions of both a symmetric band structure matrix with order $n$ and semiband width $\beta$ and a symmetric sparse matrix with order $n+m$ and $r_{i}^{U}$ numbers of off-diagonal non-zero entries in row $i$ are summarized in table 4.2 and can be found in e.g. [17]. For the Sherman-Morrison algorithm the $m$ rank-one-updates additionally lead to $m$ forward-backward substitutions and several operations. 
Table 4.1: Memory requirements for the vectorized Sherman-Morrison algorithm with $m$ updates of the stiffness matrix

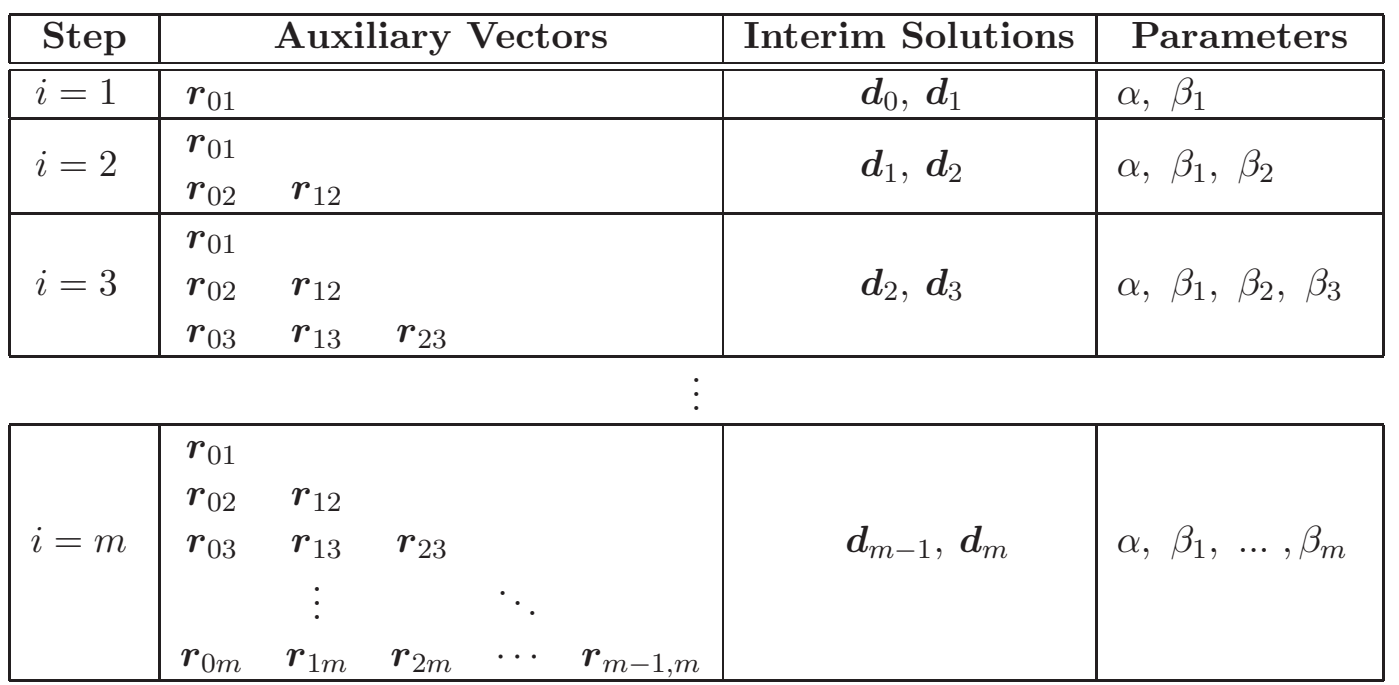

The simple example of a gas loaded cantilever beam discretized with a single row of 8 node solid shell elements shows that with an increasing number of unknowns the ShermanMorrison algorithm needs an almost equal number of operations to set up the solution vector compared to the the sparse solver (see figure 4.2). The additional operations necessary for the sequential application of the Sherman-Morrison formula do not affect the operation count remarkably - assumed that the number of rank updates is much lower than the matrix order $n$. Nevertheless it must be mentioned that additional strategies as nested dissection or minimum degree permutation are necessary to reduce the fill-in caused by the factorization of the hybrid sparse matrix. This leads to additional operations, which are not included in table 4.2. Thus for using a direct solver the derived Sherman-Morrison algorithm is at least as efficient as solving the hybrid equation system. The performance of iterative solvers for rank $m$ updated systems has not yet been investigated, but this will be part of the future work of the authors.

\section{$5 \quad$ Numerical Examples}

In the following section some numerical examples will be presented, which will cover predominantly the cases from section 3.6 and show the large variety of fluid structure interaction problems with large deformations. In the first examples the focus is on the behavior of the inner state variables and the convergence behavior during the deformation process. For this purpose relatively simple examples as proposed in [18] were chosen. Then two further examples are chosen to show the application of the derived meshless fluid structure interaction algorithms, which range from hydroforming processes in the automotive industry over gas and/or heavy fluid filled dams [10] in the field of hydraulic engineering to liquid gas loaded booster rockets [16] in the field of aerospace engineering. 
Table 4.2: Operation count for triangular factorization and foward-backward substitution with $m$ rankone-updates

\begin{tabular}{|c|c|c|}
\hline & Multiplications & Additions \\
\hline $\begin{array}{l}\text { Sherman- } \\
\text { Morrison }\end{array}$ & $\begin{array}{c}\frac{1}{2} \beta(\beta+3) n-\frac{1}{3} \beta^{3}-\beta^{2}-\frac{2}{3} \beta \\
+(m+1)\left[(2 \beta+1) n-\beta^{2}-\beta\right] \\
+m(3 n+2) \\
+\sum_{i=1}^{m-1}(m-i)(2 n+1)\end{array}$ & $\begin{array}{c}\frac{1}{2} \beta(\beta+1) n-\frac{1}{3} \beta^{3}-\frac{1}{2} \beta^{2}-\frac{1}{6} \beta \\
+(m+1)\left[2 \beta n-\beta^{2}-\beta\right] \\
+m(3 n-1) \\
+\sum_{i=1}^{m-1}(m-i)(2 n-1)\end{array}$ \\
\hline Sparse symm. & $\begin{array}{c}\sum r_{i}^{U}\left(r_{i}^{U}+3\right) / 2 \\
+n+m+2 \sum r_{i}^{U}\end{array}$ & $\sum r_{i}^{U}\left(r_{i}^{U}+1\right) / 2+2 \sum r_{i}^{U}$ \\
\hline
\end{tabular}

\subsection{Pneumatic multi chamber system}

The first example, see also [18], is to show the effect of the volume consideration first on the interaction between two pressurized chambers and second the stiffening effect of a pressurized chamber. The structure (geometric data: length $l=30 \mathrm{~mm}$, width and height $b=h=2.5 \mathrm{~mm}$, thickness $t=0.1 \mathrm{~mm}$; material data: St.-Venant Kirchhoff material, Young's modulus $E=0.75 \cdot 10^{5} \mathrm{~N} / \mathrm{mm}^{2}$, Poisson ratio $\nu=0.3$ ) is initially loaded with two different gas pressures in each chamber: the left chamber is pressurized with $p_{0}^{g, l}=0.01 \mathrm{bar}$ and the right one with $p_{0}^{g, r}=1.0 \mathrm{bar}$. Subsequently both (almost rigid) ends of the cylinder are loaded by torsion, with a prescribed final rotation of $\varphi_{\text {end }}=45^{\circ}$ and to prevent major buckling problems with a prescribed final axial displacement of $u_{e n d}=1.25 \mathrm{~mm}$ - see also figure 5.1a). The uniform FE mesh for the thin containment walls consists of 2820 solid shell elements [14]. As depicted in figure 5.1b) the chamber with low pressure is deforming heavily whereas the chamber with the high pressure shows almost no deformation at all and nearly behaves as a rigid body, which is rotating only. The stiffening effect of the internal pressure is in comparison to the empty multi chamber system - see figure 5.1c) - clearly visible. In figure 5.2 the correlation between the inner state variables $\bar{v}_{t}^{g}$ and $\Delta p_{t}^{g}$ via the adiabatic state equation (2.8) is depicted. The relative volume decrease (to the power of $\kappa$ ) is reciprocal to the relative pressure increase. Due to the stiffening effect of the chamber with high pressure the deformation affects only the left chamber, where a pressure increase of a factor of 6 is found. The pressure in the right chamber remains almost constant throughout the computation. Very small rotation increments $\Delta \varphi$ already lead to relatively large shell deflections. Figure 5.2 also shows this effect: Starting with load step $\varphi=2.5^{\circ}$ both gas volumes change with a high rate. Only 4-5 iterations are usually necessary to reach the required tolerance of $t o l=1 \cdot 10^{-12}$, see figure 5.3 a) and c). An exception is load step 5 , with $\varphi=2.5^{\circ}$, where 9 iterations are necessary - see figure $5.3 \mathrm{~b}$ ) - because in this state the system is close to buckling.

\subsection{Large deformation process of hydrostatically loaded box}

This example, presented in slightly different form in [20], is chosen to show the interaction of large deformations with the hydrostatic pressure loading and to illustrate how the transition from the statically determined system in [20] with a free fluid surface $\Gamma_{t}^{o} \neq 0$ 


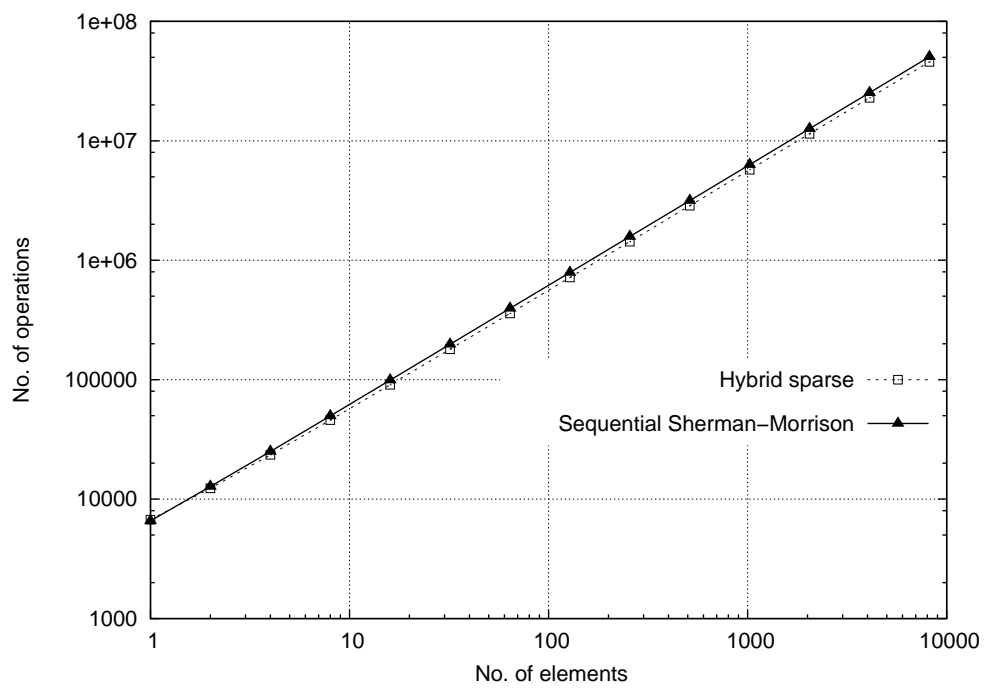

Fig. 4.2: Cantilever beam under pressure: comparison of operation count for solving the equations for different solution algorithms

to a statically undetermined system of a completely fluid filled system $\left(\Gamma_{t}^{o}=0\right)$ can be achieved. An elastic hollow hexahedral box structure (geometric data: length $l=30 \mathrm{~mm}$, width and height $b=h=5 \mathrm{~mm}$, thickness $t=0.1 \mathrm{~mm}$; material data: St.-Venant Kirchhoff material - small strains, Young's modulus $E=0.75 \cdot 10^{5} \mathrm{~N} / \mathrm{mm}^{2}$, Poisson ratio $\nu=0.3$ ) filled with a heavy fluid (initial density $\rho_{0}=1.0 \cdot 10^{-6} \mathrm{~kg} / \mathrm{mm}^{3}$ ) is loaded by torsion $\varphi_{\text {end }}=45^{\circ}$ and tension $u_{\text {end }}=1.25 \mathrm{~mm}$ (see figure 5.4). The base and top plates are (in contrast to the walls) modeled as almost rigid bodies to avoid snap through behavior during the deformation. Further on, it is assumed that a gas pressure exchange between the box and the environment is guaranteed throughout the "filling " process until there is no gas left in the box to finally achieve a purely hydrostatic loading. At the beginning of the analysis the box is partially fluid filled with a hydrostatic height of $x^{o}=24 \mathrm{~mm}$, leading to the initial hydrostatic pressure distribution depicted in figure 5.4 a) with a free fluid surface of $\Gamma^{o}=25 \mathrm{~mm}^{2}$. As shown in figure 5.6 a) and b) the deformation leads to a decreasing volume of the shell structure and thus an increase of the water level, because the basic assumption of partially fluid filled systems without gas loading/pressure is the conservation of the fluid volume $\delta \bar{v}^{f}=0$. The volume conservation can also be seen in figure 5.7 for the load steps $\varphi=0 . .16^{\circ}$. With ongoing deformation the free fluid surface also changes, reaching a minimum of $\Gamma_{t}^{o}=21.5 \mathrm{~mm}^{2}$ when the water level reaches the maximum necking point of the box at a vertical coordinate of $x_{3}=25.5 \mathrm{~mm}$. A further rise of $x^{o}$ yields again an increase of $\Gamma_{t}^{o}$ as the fluid level approaches the undeformable top plate. If the deformation continues further the fluid level reaches the maximum $x_{3}$-coordinate of the structure, leading to a statically undetermined system. Any further deformation of the structure can now only be computed considering the volumetric deformation of the fluid, which means the algorithm has to switch from an incompressible heavy fluid with free fluid surface to a container fully filled with a compressible heavy fluid without free fluid surface. A proper parameter to connect the two cases is the absolute hydraulic height $x^{o}$. At this level the fluid pressure $p_{t}^{f}$ is always identical zero (see figure 5.5).

$$
p_{t}^{f}\left(\boldsymbol{x}_{t}^{o}\right)=0
$$




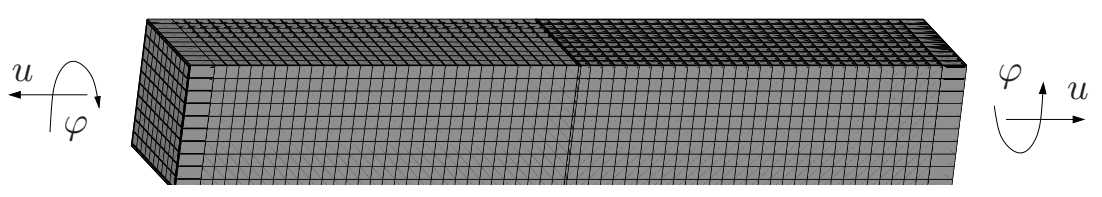

a) undeformed 2 chamber system

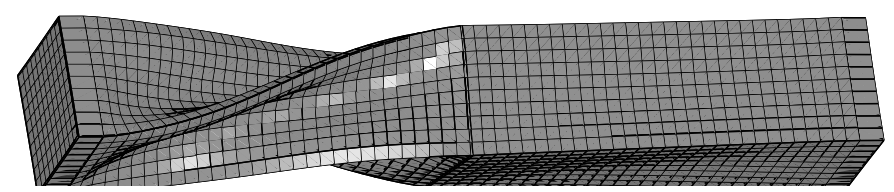

b) deformed system with low and high pressure chamber

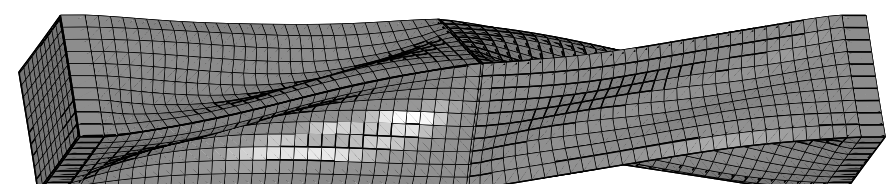

c) deformed system with two empty chambers

Fig. 5.1: Multi (two) chamber system under torsion $\varphi$ and tension $u$

According to equation (3.28) for a compressible heavy fluid with stiffness parameters $\bar{\alpha}=1$ and $\bar{\beta}=0$ the fluid pressure $p_{t}^{f}$ at any point can be decomposed into the pressure $p_{t}^{c}$ in the center of gravity $\boldsymbol{x}_{t}^{c}$, the position dependent pressure term $p_{t}^{x}$ and the pressure of the volume compression $p_{t}^{k f}$.

$$
p_{t}^{f}(\boldsymbol{x})=p_{t}^{c}-p_{t}^{x}-p_{t}^{k f}
$$

Using condition (5.1) in (5.2) yields along with

$$
x_{t}^{o}=\frac{\boldsymbol{g}}{|\boldsymbol{g}|} \cdot \boldsymbol{x}_{t}^{o}
$$

and $\boldsymbol{x}_{t}=\boldsymbol{x}_{t}^{o}$ the absolute hydraulic height for a compressible heavy fluid:

$$
\begin{aligned}
p_{t}^{f}\left(\boldsymbol{x}_{t}^{o}\right) & =p_{t}^{c}-p_{t}^{x}\left(\boldsymbol{x}^{o}\right)-p_{t}^{k f}=0 \\
\Leftrightarrow \quad x_{t}^{o} & =\frac{1}{\rho_{t}|\boldsymbol{g}|}\left(p_{t}^{c}-p_{t}^{k f}\right) .
\end{aligned}
$$

Thus the computation the deformation of the fluid filled box can be continued beyond the full state, when the fluid reaches the top plate, by considering the volume compression of the fluid using in this example a bulk modulus of $K=5.0 \mathrm{~N} / \mathrm{mm}^{2}$. As shown in figure $5.6 \mathrm{~b}$ ) for a torsion angle of $\varphi>18^{\circ}$ the absolute hydraulic height exceeds the maximum box height $\left(\Gamma_{t}^{o}=0\right)$ and already fairly small deformations - respectively a small volume compression - lead to a very high internal pressure and thus a strongly increasing hydraulic height.

The increasing volume compression, starting from $\varphi>18^{\circ}$ is directly correlated with an increase of the density of the fluid due to the mass conservation, see figure 5.7. In figure 5.8 


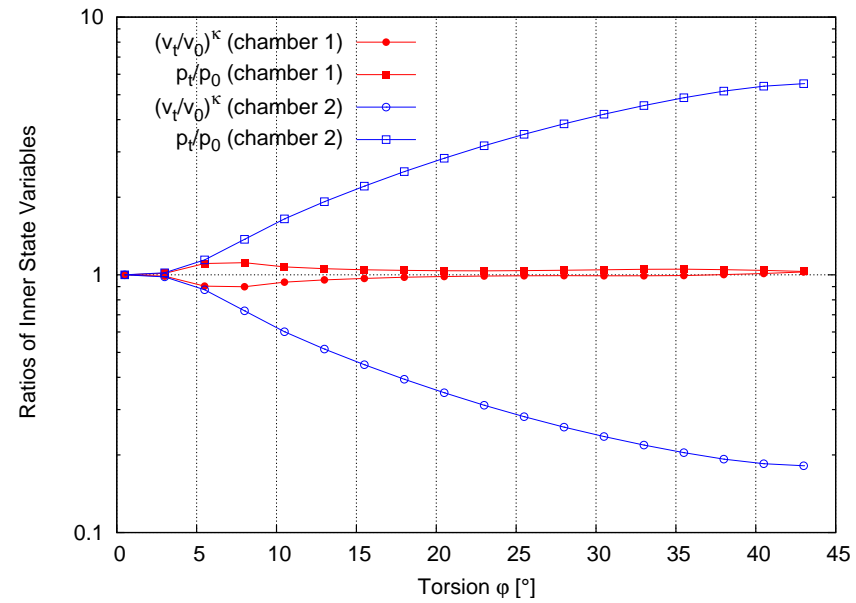

Fig. 5.2: Two chamber problem: Correlation between volume change and pressure change for each chamber during the deformation process - chamber 1: high pressure, chamber 2: low pressure

a)

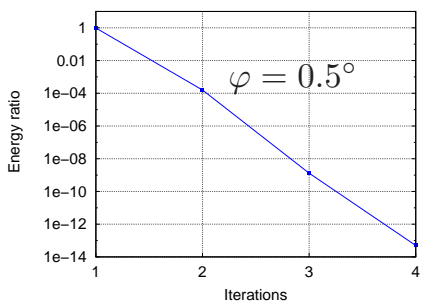

b)

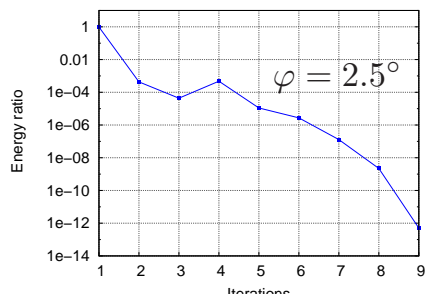

c)

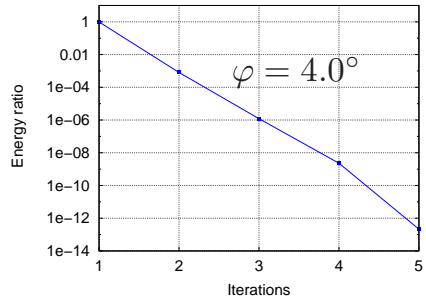

Fig. 5.3: Two chamber problem: Convergence behavior

the convergence behavior during the last 4 load steps is depicted. For the regular system only about 3 iterations are necessary to reach the tolerance of $t o l=10^{-14}$ in the energy ratio $\Delta E / E_{0}$, whereas a fairly large number of 32 iterations was necessary to manage the mass conserving transition from the incompressible fluid to the compressible fluid at load step $\varphi=18^{\circ}$. A coarse estimation of the condition number of the system matrix by the ratio of its largest and lowest diagonal entry is depicted in figure 5.9. The condition number remains throughout the computation time at a value between $1000-2000$, except for two regions $\left(\varphi \approx 5^{\circ}\right.$ and $\left.\varphi \approx 14^{\circ}\right)$, where it rises to a maximum of $2 \cdot 10^{5}$. This is due to the fact, that in these states the lowest diagonal entry (as an approximation for the lowest eigenvalue) falls down to about 100, which indicates an almost kinematical system. In this case the structure is close to buckling.

\subsection{Hydroforming of thin metal sheet}

As a typical example for fluid loaded shell structures the hydroforming of a thin metal sheet is chosen, where the uniformly distributed fluid pressure guarantees a more homogeneous thickness throughout the finally deformed part. In addition this example illustrates the versatility of the derived algorithm, because it can be applied after previous other loadings and can be combined with other conventional algorithms like structural contact and nonlinear material behavior in this example. In fig. 5.10 a) a simple sketch of the hydroforming 


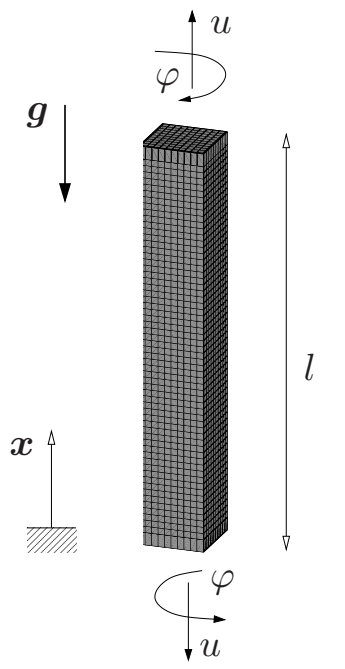

a)

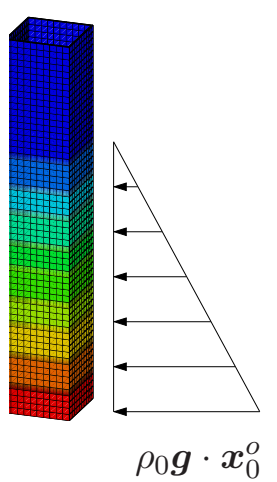

b)

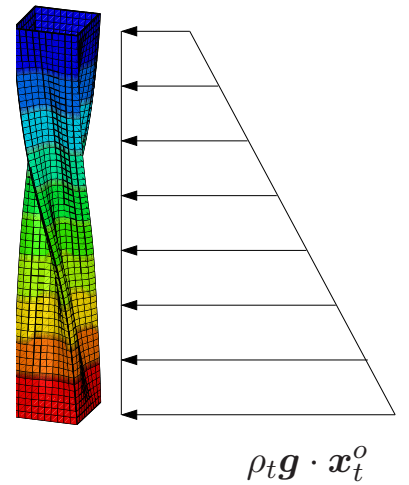

Fig. 5.4: Closed hexahedral box under torsion $\varphi$ and tension $u$ : Hydrostatic pressure distributions in a) initial state and b) final state

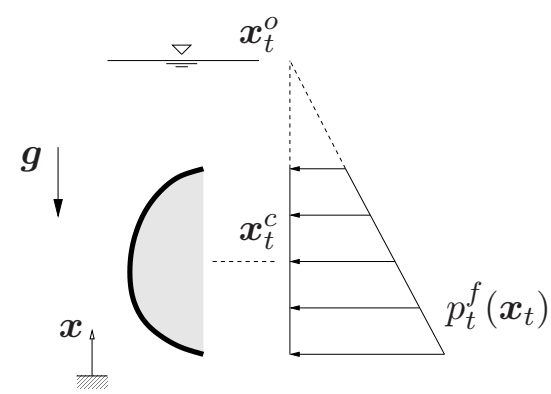

Fig. 5.5: Absolute hydraulic height for a completely fluid filled system

process is shown, where by a displacement controlled piston (stroke $v_{\text {piston }}=\Delta u 1800 \mathrm{~mm}^{2}$ ) fluid pressure is applied on the metal sheet. The pressure is further increased until the final deformation - see also fig. $5.10 \mathrm{~b}$ ) - is reached. The metal sheet has a length of $l=100 \mathrm{~mm}$, a width of $b=20 \mathrm{~mm}$ and a thickness $t=0.05 \mathrm{~mm}$. The chosen material model for the metal sheet is a hyperelastic-viscoplastic material law with isotropic hardening (bulk modulus $K_{m}=1.75 \cdot 10^{5} \mathrm{~N} / \mathrm{mm}^{2}$, shear modulus $\mu=8.077 \cdot 10^{5} \mathrm{~N} / \mathrm{mm}^{2}$, yield stress $\sigma_{y}=160 \mathrm{~N} / \mathrm{mm}^{2}$ and hardening modulus $H=200 \mathrm{~N} / \mathrm{mm}^{2}$ ). The contact along the matrix surface is simulated by a pure penalty scheme. The computation was performed at a quarter of the full metal sheet exploiting the symmetry of the structure. The model was discretized with 46 solid shell elements [14]. Using a bulk modulus of $K_{f}=5.0 \mathrm{~N} / \mathrm{mm}^{2}$ for the fluid results in a relatively stiff behavior of the fluid. In figure 5.11 a) remarkable volume change of the fluid is found beyond a piston displacement of $\Delta u=1.5 \mathrm{~mm}$. From this point on the metal sheet, by then almost fully plastified, is in contact with the matrix and further deflections are only possible under high pressures with the consequence of high volume compression. In the first load steps only 3 iterations are necessary to reach the convergence tolerance level - see figure 5.12 a), whereas in the last load steps - see figure $5.12 \mathrm{~b}$ ), where almost each element is in contact with the matrix up to ten iterations are needed. This is due to the fact that in the last load steps a very stiff structure is present and 


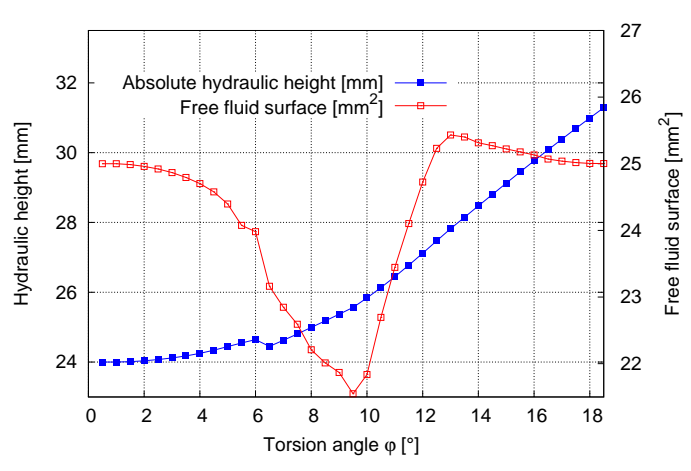

a) incompressible heavy fluid

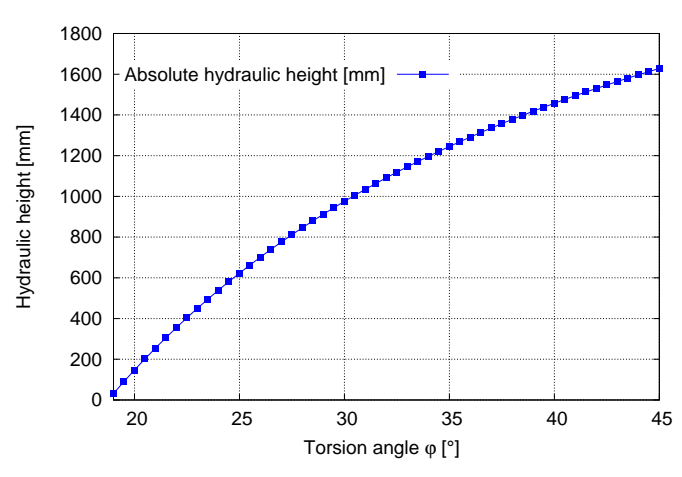

b) compressible heavy fluid

Fig. 5.6: Closed hexahedral box under torsion and tension: Development of fluid variables during loading - a) incompressible heavy fluid (free fluid surface $>0$ ) and b) compressible heavy fluid (free fluid surface $=0$ )

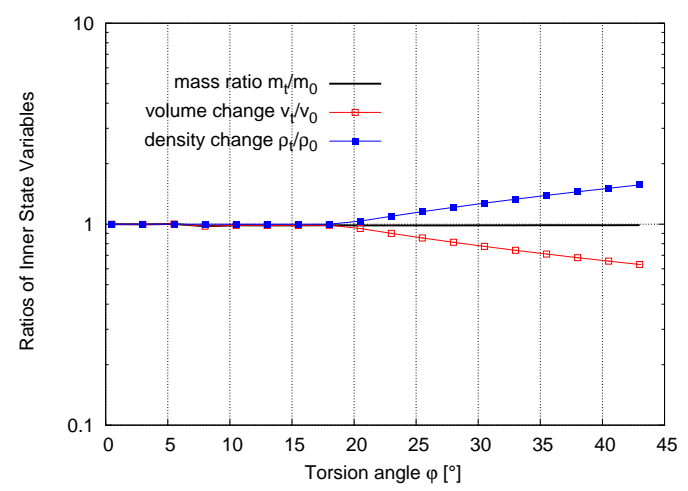

Fig. 5.7: Closed hexahedral box under torsion and tension: Development of inner state variables during loading time

already small deflections caused by the contact penalty forces induce high internal fluid pressures whereas on the other hand the high fluid pressure leads to penetrations causing fairly high penalty forces again. Therefore it is advisable to use small load increments then.

\subsection{Inflation and hydrostatic loading of an inflatable dam}

The inflation and hydrostatic loading of a rubber dam under several boundary conditions will serve as another real world example for a multi chamber system with varying loading conditions. Figure 5.13 illustrates such an inflatable dam under hydrostatic loading; hydrodynamic effects will not be considered in these examples. For a discussion of the general application of such dams we refer to [10]. The rubber dam with a geometry simplified for the analysis in this contribution consists of two membrane parts, which are hot vulcanized along one edge resulting in the typical deflection fin, which later in the real world is helpful to reduce vibrations. Along the other edge the two membrane layers are clamped and anchored to the ground. The most important task of controlled dams is the adjustment of the water retention level or the dam height, which allows to take control of the discharge. In the case of inflatable dams the dam height $h$ can be simply regulated 


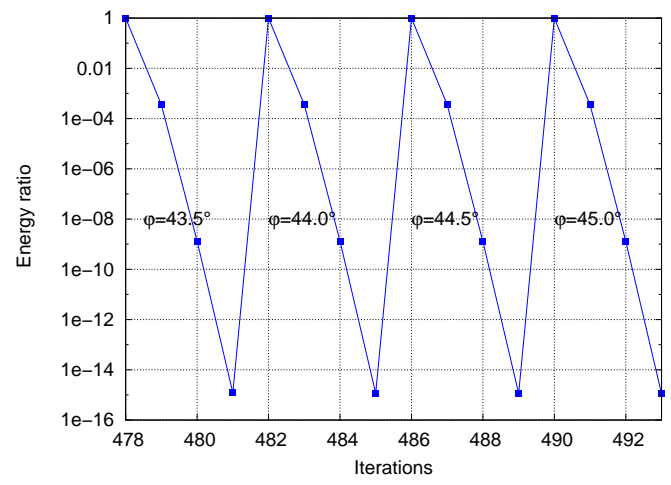

Fig. 5.8: Closed hexahedral box under torsion and tension: Convergence behavior in last 4 load steps

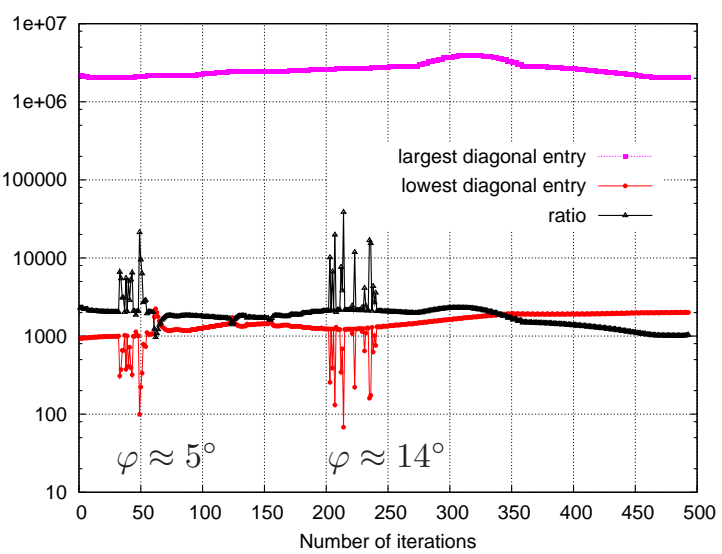

Fig. 5.9: Hydrostatic loaded box: Estimation of condition number by diagonal entries

by the internal gas pressure. Analytical solutions for different load cases can be found in [3] assuming pure membrane boundary conditions and bending free joining of the membrane parts. The following computations to simulate the development of the dam height during the loading process are performed in several steps: First the membrane structure is deployed by increasing the internal pressure. Then the head and bottom water levels will be raised. The membranes for the dam (scale 1:10 in the model structure built in [10]) have a thickness of $t=2 \mathrm{~mm}$ and a total circumference of about $l_{S}=850 \mathrm{~mm}$, see figure 5.13. The material data with Young's modulus $E=60 \mathrm{~N} / \mathrm{mm}^{2}$ and Poisson ratio $\nu=0.4$ correspond to a fiber reinforced rubber membrane. In this special load case with head water loading, bottom water loading and an interior gas filling a 3-chamber system is the appropriate analysis model. Although the head and bottom water levels are not affected by the deformation of the dam, which means that the rank updates of the system matrix result only from the gas filled chamber, the normal change parts and the residual vectors for all 3 chambers have to be considered. Further, boundary effects (e.g. wrinkling of membrane parts) in the axial direction of the dam, resulting from a real world construction process at the boundary, are neglected in the current model. Therefore we can restrict the investigation to a $2 \mathrm{D}$ model of the cross section discretized with 66 so-called solid-shell elements [14] with linear shape functions and ANS/EAS enhancements for the kinematics. In future publications the simulation of a real world fully $3 \mathrm{D}$ model of the 


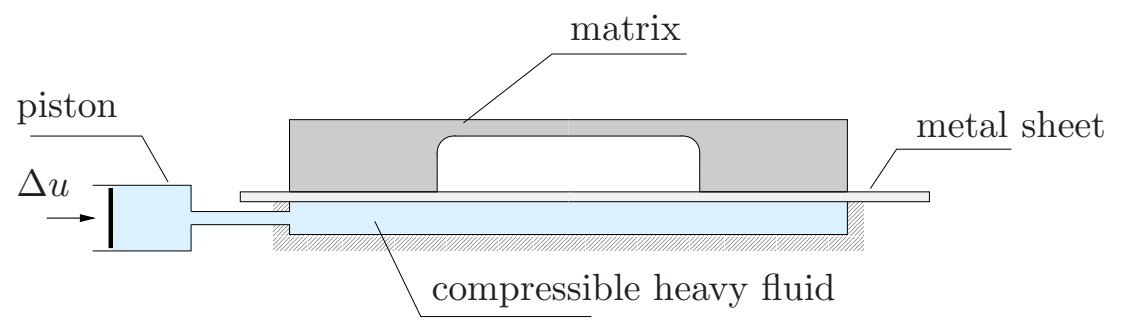

a) sketch of hydroforming process

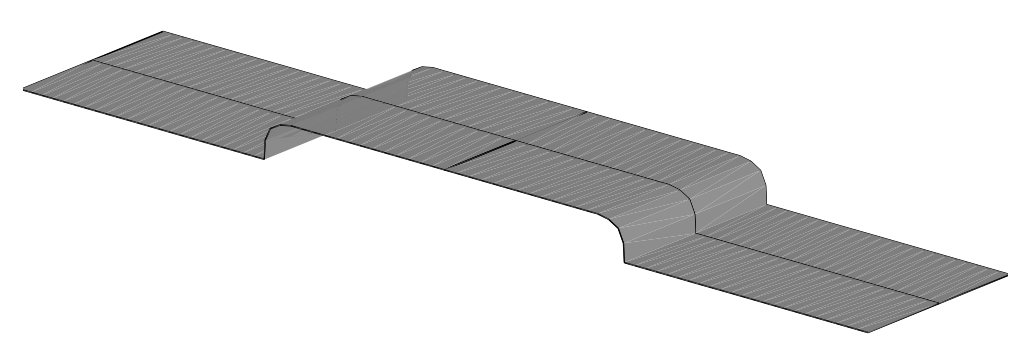

b) metal sheet after deformation process

Fig. 5.10: Hydroforming of a metal sheet against a matrix controlled by the motion of a piston

dam will be presented, considering also the specific contact and support conditions at the boundaries leading often to wrinkling.

\subsubsection{Clamping along a single edge — only gas filling: load case I}

The first possible layout of an inflatable dam has a single clamped edge on the left, see figure 5.14a). The body of the dam is in contact (penalty formulation) with the ground. The dam is first pressurized until "load step" 10 , when the maximum dam height of $h=220 \mathrm{~mm}$ is reached, assuming static loading. Then the head water level is steadily increased, which in the end leads to a kind of rolling motion of the dam, clearly visible in the decrease of the dam height of about 10\%, see figure 5.14b). From load step 20 on the downstream water level is slowly raised. This additional loading by the bottom water leads to an undesired buoyancy of the dam body, because the membrane is only clamped along the left edge. A possible way to overcome this buoyancy (besides clamping two edges along the dam body) is presented in the next subsection.

\subsubsection{Clamping along a single edge — gas and interior heavy fluid filling: load case II}

In order to avoid the upward buoying of the dam body its interior is now filled with heavy fluid in addition to the gas filling, see figure 5.15a). In figure 5.15b) the development of the dam height during the loading process is given: In this case the interior fluid support reduces the dam height by a few percent. The last load increments of head water filling then again lead to a sudden decrease of about $10-15 \%$ of the original height. But in contrast to the first example the plateau of the dam height curve shows that due to interior fluid support no upward buoying effects appear. 


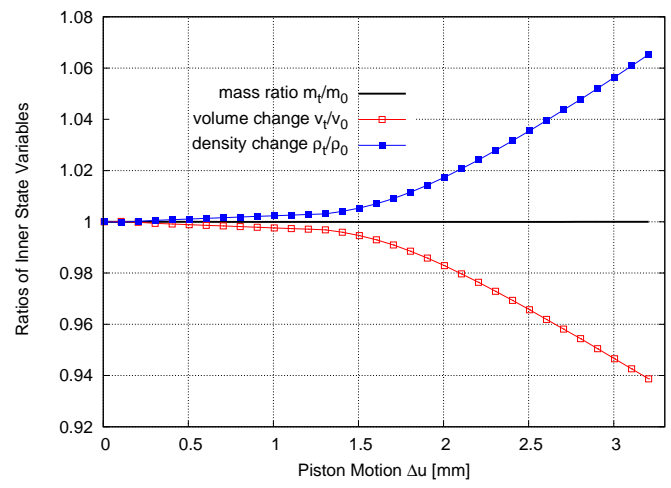

Fig. 5.11: Hydroforming of a metal sheet: Correlation between density change and volume change during deformation process

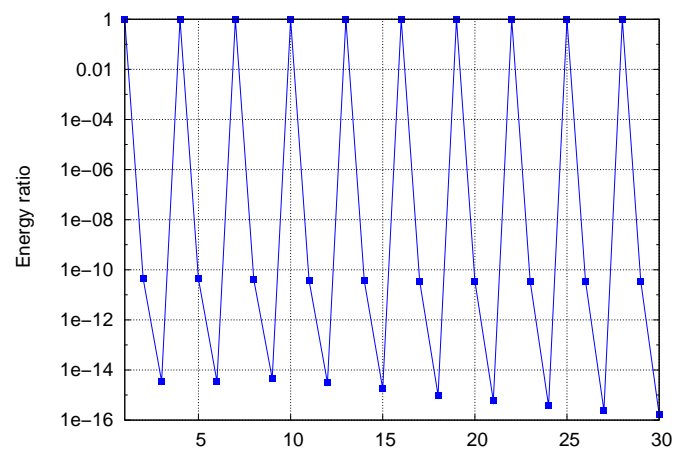

a) No. of iterations in first load steps

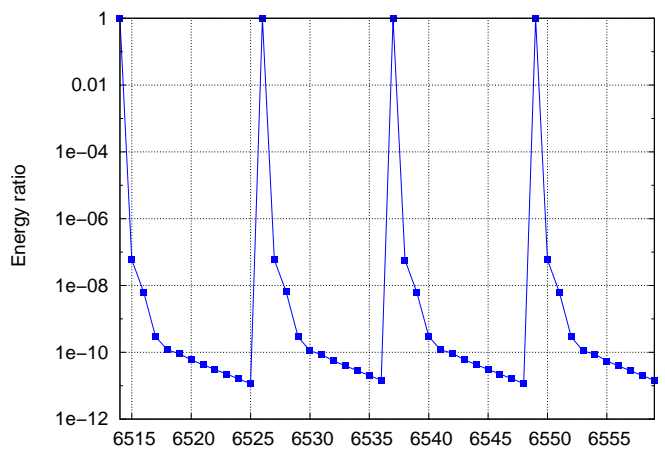

b) No. of iterations in last load steps

Fig. 5.12: Hydroforming of a metal sheet: Convergence behavior during loading process in various stages

\subsection{Discussion of volume coupling terms}

The case of pneumatic membrane structures featuring a volume dependent gas support has already been part of several investigations, see [5], [6], [8] and [19]. As e.g. demonstrated in [6] for the inflation of a tube, considering the rank-one update of the stiffness matrix due to volume coupling led to a significantly higher convergence rate as it did without. Berry and Yang [5] investigated a snap through problem of a curved shell under pressure. Neglecting the additional coupling terms in the stiffness computation in their particular case led to the typical snap through load deflection curve, which can (statically) only be solved by using path following methods, e.g. by an arc length method. Considering the volume coupling of the internal gas pressure the structural behavior was totally different. The load deflection curve had always a positive slope, because the rank update led to a stabilization of the global stiffness matrix (see also [13]) and thus enabled the use of standard Newton-schemes for the solution process. However, in general it is not always necessary to take into account all volume coupling terms.

Having first a closer look at the global system matrix $\boldsymbol{K}^{\text {sys }}$ of a pure gas loaded/supported system yields with equations (3.44) and (3.45) and table 3.1:

$$
\boldsymbol{K}^{s y s}=\boldsymbol{K}^{e l}+\boldsymbol{K}^{g}+\alpha_{t} \boldsymbol{a} \boldsymbol{a}^{T}
$$




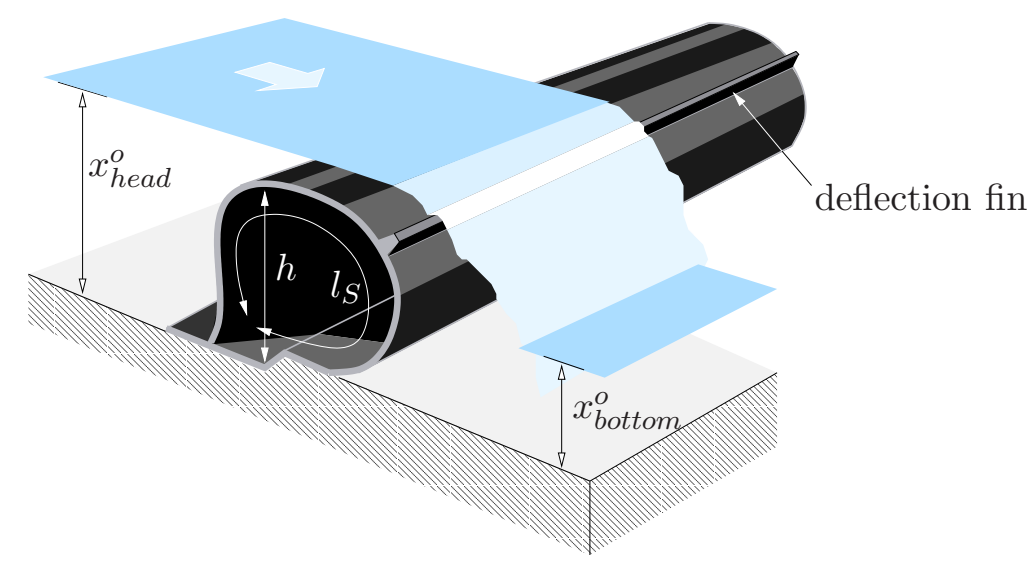

Fig. 5.13: Sketch of inflated rubber dam under head and bottom water loading

a)

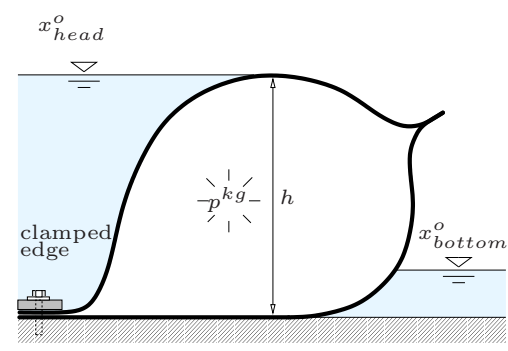

b)

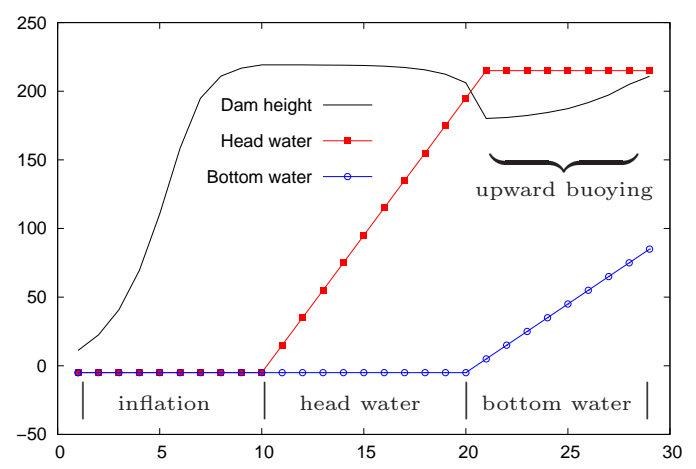

Fig. 5.14: Inflation and loading of rubber dam - gas filling: load case I - a) layout of the dam and b) loading process and development of dam height

To estimate the influence of the volume coupling on the global stiffness matrix it is necessary to identify the dominating variables in each term. The bending and membrane stiffnesses are the dominating variables of $\boldsymbol{K}^{e l}$. In addition nonlinear geometric stiffness effects arising from large deformations $\varepsilon\left(\boldsymbol{u}_{t}\right)$ have to be considered.

$$
\boldsymbol{K}^{e l}=\boldsymbol{K}^{e l}\left(E I, E A, \boldsymbol{u}_{t}\right)
$$

The nonlinear, so-called load-stiffness part $\boldsymbol{K}^{g}$ is primarily dependent on the discrete area $d \Gamma$, the pressure $p_{t}^{k g}$ and the current displacement field $\boldsymbol{u}_{t}$ of each wetted finite element, see also equation (3.26).

$$
\boldsymbol{K}^{g}=\boldsymbol{K}^{g}\left(d \Gamma, p_{t}^{k g}, \boldsymbol{u}_{t}\right)
$$

The final volume coupling term shows only a dependence on the discrete area $d \Gamma$ of each wetted finite element, see equation (3.41),

$$
\boldsymbol{a}=\boldsymbol{a}(d \Gamma)
$$


a)

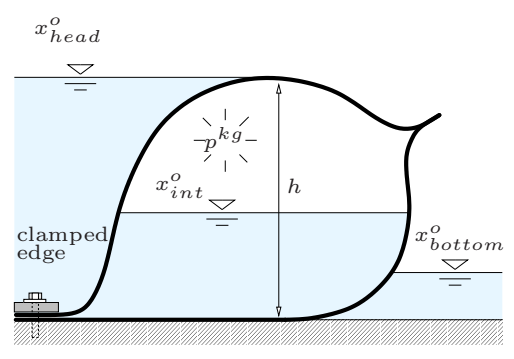

b)

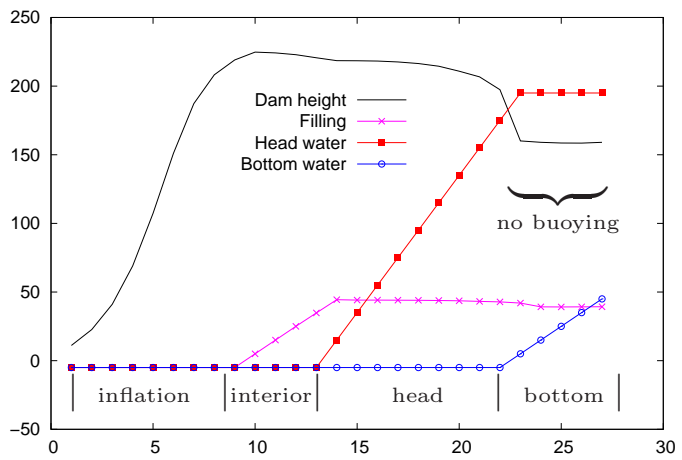

Fig. 5.15: Filling/inflation and loading of rubber dam - gas and fluid filling: load case II - a) layout of the dam and b) loading process and development of dam height

and the pressure volume gradient $\alpha_{t}$, which is a function of the gas pressure $p_{t}^{k g}$, the gas volume $\bar{v}_{t}^{g}$ and the isentropic constant $\kappa$, see equation (2.18).

$$
\alpha_{t}=\kappa \frac{p^{k g}}{\bar{v}_{t}^{g}}
$$

Three factors are determining the relevance of the volume coupling term on the solution:

- the bending stiffness $E I$ of the shell element

- the pretensioning of the shell due to pressure and large deformations $\varepsilon\left(\boldsymbol{u}_{t}\right)$

- the pressure volume gradient $\alpha_{t}$

For relatively small control volumes $\bar{v}_{t}^{g}$ in an almost membrane-like structure with negligible bending stiffness, the volume coupling dominates the solution, as already observed in [5] and [6], affecting also the convergence of the Newton scheme positively. Then the pressure volume gradient becomes rather large and guarantees the system matrix to be positive definite throughout the computation. For stiff structures (either obtained by pretensioning or due to large bending stiffness $E I$ ) and/or very large control volumes the pressure volume gradient is negligible compared to the entries in the stiffness matrix $\boldsymbol{K}^{e l}$ and in $\boldsymbol{K}^{g}$ and therefore the solution process and convergence is only marginally affected by the volume coupling, see [13]. Thus the decision to include or neglect the additional rank-one updates depends on the specific problem and/or the specific state of a problem.

For a system loaded/supported with a compressible fluid the rank-one updated stiffness matrix (see equations (3.44) and (3.45) and table 3.1) - neglecting for simplicity the gravity effect - becomes

$$
\boldsymbol{K}^{s y s}=\boldsymbol{K}^{e l}+\boldsymbol{K}^{f}+\beta_{t} \boldsymbol{b} \boldsymbol{b}^{T}
$$

and thus the same general arguments as for the gas loaded/supported system are valid. In this case the important factor is the pressure volume gradient

$$
\beta_{t}=\frac{K}{\bar{v}_{0}^{f}}
$$

of the fluid, see equation (2.19). Small initial control volumes $\bar{v}_{0}^{f}$ and/or a large bulk modulus $K$ lead in principle, for thin-walled and membrane-like structures in identical fashion 
as for the gas loading/support to a domination of the volume coupling terms. In addition we have to note that the factor $\beta_{t}$ is usually much larger than $\alpha_{t}$ with the conclusion that for small enclosed volumes the volume coupling should always be considered. In particular, for stability analysis of structures containing fluids with substantial filling the volume coupling is important.

\section{Conclusions}

Based on the contributions of [6], [18], [19], [20] and [21], where algorithms for volume dependent gas and fluid loadings/fillings were given, in this contribution a general description of all possible load cases concerning static fluid structure interaction is presented. Starting with a total energy approach for the fluid and gas terms and introducing special stiffness parameters a general description was derived. The fluid-gas-structure-interaction was described via boundary integrals of the parts of the structure wetted by fluid and/or gas. A consistent linearization of the volume dependent terms and the normal change parts along with the consideration of physically realistic boundary conditions led to symmetric system matrices proving the conservativeness of the problem. Varying the values of the stiffness parameters the general case with flexible gas and flexible fluid as well as all specific cases: purely gas filling, gas plus incompressible fluid, only incompressible fluid and only compressible fluid as partially shown in [19], [20] and [21] could be developed. Further both, the extension to multi chamber problems and the combination of fluid and gas loading in single chambers were presented. An efficient solution algorithm for this kind of problems can be found using the Sherman-Morrison formula in a sequential fashion, which allows to avoid the direct inversion of the dyadic updated and at least section-wise fully populated system matrix, which in addition may be badly conditioned in case of fluid support with a nearly incompressible fluid. A further focus of the authors is currently on the stability investigation of gas/fluid filled or loaded multi chamber systems [13], especially inflatable dams, which often suffer from instability problems.

\section{Solution algorithm for a dyadic updated system matrix}

For all finite elements, which have a surface contacting the fluid or the gas coupling vectors are generated. As shown above in equation (3.69) the dyadic rank updates with the coupling vectors lead for each chamber to a fully occupied section of the system matrix. To bypass the solution with such an almost fully populated system matrix a general scheme is presented, which is based on a sequential application of the Sherman-Morrison formula, see also [9], [18] or [25]. By introducing the abbreviations

$$
\boldsymbol{A}=\boldsymbol{K}+\sum_{l=1}^{m} \boldsymbol{x}_{l} \boldsymbol{y}_{l}^{T}, \quad \text { with } \quad \boldsymbol{K}=\boldsymbol{K}^{e l}+\sum_{i=1}^{n}\left(\boldsymbol{K}_{i}^{g}+\boldsymbol{K}_{i}^{f}\right)
$$

where the load-stiffness matrices have almost the same structure as the elastic stiffness matrices and do not lead to a different band width. The right hand side vector is given as:

$$
\boldsymbol{f}=\boldsymbol{f}^{e x}-\boldsymbol{f}^{e l}-\sum_{i=1}^{n}\left(\boldsymbol{f}_{i}^{g}+\boldsymbol{f}_{i}^{f}\right)
$$


Then the solution vector can be written as:

$$
\boldsymbol{d}=\boldsymbol{A}^{-1} \boldsymbol{f} \text {. }
$$

Looking at equation (7.1) as a modification of $\boldsymbol{A}$

$$
\boldsymbol{K}=\boldsymbol{A}-\sum_{l=1}^{m} \boldsymbol{x}_{l} \boldsymbol{y}_{l}^{T}
$$

we obtain by left-hand multiplication of (7.4) with $\boldsymbol{K}^{-1}$ and subsequent right-hand multiplication with $\boldsymbol{A}^{-1} \boldsymbol{f}$ a recursive form for the solution vector.

$$
\boldsymbol{d}=\boldsymbol{K}^{-1} \boldsymbol{f}-\boldsymbol{K}^{-1} \sum_{l=1}^{m} \boldsymbol{x}_{l} \boldsymbol{y}_{l}^{T} \boldsymbol{d} .
$$

\subsection{Solution for a rank 3 update of $K$}

In order to derive a general scheme for this recursive form, the solution is shown for a stiffness matrix $\boldsymbol{K}$ with a $(m=3)$-rank update. Afterwards the general rule for the computations in an arbitrary solution step $i$ is set up. A special focus is here on a consequent vectorization of the equations to avoid storing fully populated stiffness matrices or fully populated sections of the stiffness matrix.

\subsubsection{Starting point: recursive step $i=0$}

To reduce the expressions in the following steps the notations $\boldsymbol{d}_{0}=\boldsymbol{K}^{-1} \boldsymbol{f}$ and $\boldsymbol{\Psi}_{0}=-\boldsymbol{K}^{-1}$ are introduced. Thus from equation (7.5) a form is obtained, which gives more insight into the triple update of the conventional solution vector $\boldsymbol{d}_{0}$ with further unknown vectors resulting from the coupling vectors.

$$
\boldsymbol{d}=\boldsymbol{d}_{0}+\boldsymbol{\Psi}_{0} \sum_{l=1}^{n=3} \boldsymbol{x}_{l} \boldsymbol{y}_{l}^{T} \boldsymbol{d} \quad(\text { Step } i=0) \text {. }
$$

Starting from equation (7.6) we can proceed with the first real recursive step:

\subsubsection{Recursive step $i=1$}

Separating the summand with index $l=i=1$ from the sum in equation (7.6) gives us

$$
\boldsymbol{d}=\boldsymbol{d}_{0}+\Psi_{0} \boldsymbol{x}_{1} \boldsymbol{y}_{1}^{T} \boldsymbol{d}+\Psi_{0} \sum_{l=2}^{m=3} \boldsymbol{x}_{l} \boldsymbol{y}_{l}^{T} \boldsymbol{d} .
$$

By using the auxiliary vector

$$
\boldsymbol{r}_{01}=\Psi_{0} \boldsymbol{x}_{1}
$$

we can abbreviate the solution to

$$
\boldsymbol{d}=\boldsymbol{d}_{0}+\boldsymbol{r}_{01} \boldsymbol{y}_{1}^{T} \boldsymbol{d}+\mathbf{\Psi}_{0} \sum_{l=2}^{m=3} \boldsymbol{x}_{l} \boldsymbol{y}_{l}^{T} \boldsymbol{d}
$$

A further multiplication of (7.9) from the left side with the rank vector $\boldsymbol{y}_{1}^{T}$ then leads to

$$
\boldsymbol{y}_{1}^{T} \boldsymbol{d}=\boldsymbol{y}_{1}^{T} \boldsymbol{d}_{0}+\boldsymbol{y}_{1}^{T} \boldsymbol{r}_{01} \boldsymbol{y}_{1}^{T} \boldsymbol{d}+\boldsymbol{y}_{1}^{T} \boldsymbol{\Psi}_{0} \sum_{l=2}^{m=3} \boldsymbol{x}_{l} \boldsymbol{y}_{l}^{T} \boldsymbol{d}
$$


Rewriting this equation results in:

$$
\begin{aligned}
& \boldsymbol{y}_{1}^{T} \boldsymbol{d}-\boldsymbol{y}_{1}^{T} \boldsymbol{r}_{01} \boldsymbol{y}_{1}^{T} \boldsymbol{d}=\boldsymbol{y}_{1}^{T} \boldsymbol{d}_{0}+\boldsymbol{y}_{1}^{T} \boldsymbol{\Psi}_{0} \sum_{l=2}^{m=3} \boldsymbol{x}_{l} \boldsymbol{y}_{l}^{T} \boldsymbol{d} \\
& \Leftrightarrow \quad\left(1-\boldsymbol{y}_{1}^{T} \boldsymbol{r}_{01}\right) \boldsymbol{y}_{1}^{T} \boldsymbol{d}=\boldsymbol{y}_{1}^{T} \boldsymbol{d}_{0}+\boldsymbol{y}_{1}^{T} \boldsymbol{\Psi}_{0} \sum_{l=2}^{m=3} \boldsymbol{x}_{l} \boldsymbol{y}_{l}^{T} \boldsymbol{d}
\end{aligned}
$$

Isolating the scalar term $\boldsymbol{y}_{1}^{T} \boldsymbol{d}$ on the left hand side yields along with the parameter

$$
\beta_{1}=\left(1-\boldsymbol{y}_{1}^{T} \boldsymbol{r}_{01}\right)^{-1}
$$

the following form:

$$
\boldsymbol{y}_{1}^{T} \boldsymbol{d}=\beta_{1} \boldsymbol{y}_{1}^{T} \boldsymbol{d}_{0}+\beta_{1} \boldsymbol{y}_{1}^{T} \Psi_{0} \sum_{l=2}^{m=3} \boldsymbol{x}_{l} \boldsymbol{y}_{l}^{T} \boldsymbol{d}
$$

Reinserting (7.13) in (7.9) gives

$$
\boldsymbol{d}=\boldsymbol{d}_{0}+\boldsymbol{r}_{01}\left(\beta_{1} \boldsymbol{y}_{1}^{T} \boldsymbol{d}_{0}+\beta_{1} \boldsymbol{y}_{1}^{T} \boldsymbol{\Psi}_{0} \sum_{l=2}^{m=3} \boldsymbol{x}_{l} \boldsymbol{y}_{l}^{T} \boldsymbol{d}\right)+\boldsymbol{\Psi}_{0} \sum_{l=2}^{m=3} \boldsymbol{x}_{l} \boldsymbol{y}_{l}^{T} \boldsymbol{d}
$$

Rearranging the terms associated to the dyadic update ends the first recursive step:

$$
\boldsymbol{d}=\boldsymbol{d}_{0}+\beta_{1} \boldsymbol{r}_{01} \boldsymbol{y}_{1}^{T} \boldsymbol{d}_{0}+\left(\beta_{1} \boldsymbol{r}_{01} \boldsymbol{y}_{1}^{T} \mathbf{\Psi}_{0}+\Psi_{0}\right) \sum_{l=2}^{m=3} \boldsymbol{x}_{l} \boldsymbol{y}_{l}^{T} \boldsymbol{d}
$$

Computing the new interim solution

$$
\boldsymbol{d}_{1}=\boldsymbol{d}_{0}+\beta_{1} \boldsymbol{r}_{01} \boldsymbol{y}_{1}^{T} \boldsymbol{d}_{0}
$$

and introducing the updated auxiliary matrix

$$
\boldsymbol{\Psi}_{1}=\beta_{1} \boldsymbol{r}_{01} \boldsymbol{y}_{1}^{T} \mathbf{\Psi}_{0}+\mathbf{\Psi}_{0}
$$

then brings back the updated form of (7.6):

$$
\boldsymbol{d}=\boldsymbol{d}_{1}+\mathbf{\Psi}_{1} \sum_{l=2}^{m=3} \boldsymbol{x}_{l} \boldsymbol{y}_{l}^{T} \boldsymbol{d} \quad(\text { Step } i=1)
$$

Now we have achieved a reduction from a rank-3-update to a rank-2-update. The next two sequent steps are performed analogously to the procedure outlined above, therefore only the necessary equations will be briefly presented.

\subsubsection{Recursive step $i=2$}

Starting from the recursive equation (7.18) for the solution vector, we multiply it from the left with the rank vector $\boldsymbol{y}_{2}^{T}$. Isolating and reinserting the term $\boldsymbol{y}_{2}^{T} \boldsymbol{d}$ in equation (7.18) yields along with 2 auxiliary vectors

$$
\begin{aligned}
& \boldsymbol{r}_{02}=\boldsymbol{\Psi}_{0} \boldsymbol{x}_{2} \quad \text { and } \\
& \boldsymbol{r}_{12}=\boldsymbol{\Psi}_{1} \boldsymbol{x}_{2}=\left(\beta_{1} \boldsymbol{r}_{01} \boldsymbol{y}_{1}^{T} \boldsymbol{\Psi}_{0}+\boldsymbol{\Psi}_{0}\right) \boldsymbol{x}_{2}=\beta_{1} \boldsymbol{r}_{01} \boldsymbol{y}_{1}^{T} \boldsymbol{r}_{02}+\boldsymbol{r}_{02}
\end{aligned}
$$


and the parameter

$$
\beta_{2}=\left(1-\boldsymbol{y}_{2}^{T} \boldsymbol{r}_{12}\right)^{-1}
$$

the next recursive rule for $\boldsymbol{d}$ :

$$
\boldsymbol{d}=\left(\boldsymbol{d}_{1}+\beta_{2} \boldsymbol{r}_{12} \boldsymbol{y}_{2}^{T} \boldsymbol{d}_{1}\right)+\left(\beta_{2} \boldsymbol{r}_{12} \boldsymbol{y}_{2}^{T} \boldsymbol{\Psi}_{1}+\boldsymbol{\Psi}_{1}\right) \sum_{l=3}^{m=3} \boldsymbol{x}_{l} \boldsymbol{y}_{l}^{T} \boldsymbol{d}
$$

Because the dyadic update has now been reduced to the last remaining term, equation (7.22) can be written in more general form:

$$
\boldsymbol{d}=\boldsymbol{d}_{2}+\boldsymbol{\Psi}_{2} \boldsymbol{x}_{3} \boldsymbol{y}_{3}^{T} \boldsymbol{d}
$$

with

$$
\boldsymbol{d}_{2}=\boldsymbol{d}_{1}+\beta_{2} \boldsymbol{r}_{12} \boldsymbol{y}_{2}^{T} \boldsymbol{d}_{1}
$$

and

$$
\boldsymbol{\Psi}_{2}=\beta_{2} \boldsymbol{r}_{12} \boldsymbol{y}_{2}^{T} \boldsymbol{\Psi}_{1}+\boldsymbol{\Psi}_{1}
$$

\subsubsection{Recursive step $i=3$}

To compute the final solution the Sherman-Morrison formula is applied again. Like in the previous subsections the recursive equation (7.23) is multiplied from the left with the remaining rank vector $\boldsymbol{y}_{3}^{T}$. Isolating and reinserting the term $\boldsymbol{y}_{3}^{T} \boldsymbol{d}$ in equation (7.23) yields after the 3rd step along with the 3 auxiliary vectors

$$
\begin{aligned}
& \boldsymbol{r}_{03}=\boldsymbol{\Psi}_{0} \boldsymbol{x}_{3} \\
& \boldsymbol{r}_{13}=\boldsymbol{\Psi}_{1} \boldsymbol{x}_{3}=\left(\beta_{1} \boldsymbol{r}_{01} \boldsymbol{y}_{1}^{T} \boldsymbol{\Psi}_{0}+\boldsymbol{\Psi}_{0}\right) \boldsymbol{x}_{3}=\beta_{1} \boldsymbol{r}_{01} \boldsymbol{y}_{1}^{T} \boldsymbol{r}_{03}+\boldsymbol{r}_{03} \\
& \boldsymbol{r}_{23}=\boldsymbol{\Psi}_{2} \boldsymbol{x}_{3}=\left(\beta_{2} \boldsymbol{r}_{12} \boldsymbol{y}_{2}^{T} \boldsymbol{\Psi}_{1}+\boldsymbol{\Psi}_{1}\right) \boldsymbol{x}_{3}=\beta_{2} \boldsymbol{r}_{12} \boldsymbol{y}_{2}^{T} \boldsymbol{r}_{13}+\boldsymbol{r}_{13}
\end{aligned}
$$

and the parameter

$$
\beta_{3}=\left(1-\boldsymbol{y}_{3}^{T} \boldsymbol{r}_{23}\right)^{-1}
$$

the final rule for the computation of the solution vector

$$
\boldsymbol{d}=\boldsymbol{d}_{3}=\left(\boldsymbol{d}_{2}+\beta_{3} \boldsymbol{r}_{23} \boldsymbol{b}_{3}^{T} \boldsymbol{d}_{2}\right) .
$$

Hence, the solution with the rank- $(m=3)$-update has been reduced to the triangularization of $\boldsymbol{K}$ and 4 forward-backward substitutions plus 10 scalar products and 7 vector summations. Further more, in each step $i$ the auxiliary vectors $\boldsymbol{r}_{j i}$ with $i=1 . . m$ and $j=0 . .(i-1)$ and the parameter $\beta_{i}$ had to be computed to get the current update of the last interim solution $\boldsymbol{d}_{i-1}$. 


\section{References}

[1] O. Andrianarison and R. Ohayon. Compressibility and gravity effects in internal fluid-structure vibrations: Basic equations and appropriate variational formulations. Computer Methods in Applied Mechanics and Engineering, 195:1958-1972, 2006.

[2] O. Andrianarison and R. Ohayon. Reduced models for modal analysis of fluidstructure systems taking into account compressibility and gravity effects. Computer Methods in Applied Mechanics and Engineering, 195:5656-5672, 2006.

[3] H. O. Anwar. Inflatable dams. Journal of the Hydraulics Division, ASCE, 93:99-119, 1967.

[4] H. D. Baehr. Thermodynamik. Springer-Verlag Berlin, 2005.

[5] D. Berry and H. Yang. Formulation and experimental verification of a pneumatic finite element. International Journal for Numerical Methods in Engineering, 39:1097-1114, 1996.

[6] J. Bonet, R. D. Wood, J. Mahaney, and P. Heywood. Finite element analysis of air supported membrane structures. Computer Methods in Applied Mechanics and Engineering, 190:579-595, 2000.

[7] R. de Boer. Vektor- und Tensorrechnung für Ingenieure. Springer-Verlag Berlin, 1982.

[8] R. van Dijk, F. van Keulen, and J. Sterk. Simulation of closed thin-walled structures partially filled with fluid. International Journal of Solids and Structures, 37:60636083,2000 .

[9] J. D. Faires and R. L. Burden. Numerische Methoden. Spektrum Akademischer Verlag, 1994.

[10] M. Gebhardt. Hydraulische und statische Bemessung von Schlauchwehren. PhD thesis, Institut für Wasserwirtschaft und Kulturtechnik der Universität Karlsruhe (TH), 2006.

[11] A. George. Nested dissection of a regular finite element mesh. SIAM Journal on Numerical Analysis, 10:345-363, 1973.

[12] A. George and J. Liu. The evolution of minimum degree ordering algorithm. SIAM Review, 31:1-19, 1989

[13] M. Haßler and K. Schweizerhof. On the influence of fluid-structure-interaction on the static stability of thin walled shell structures. International Journal of Structural Stability, 7:313-335, 2007.

[14] R. Hauptmann and K. Schweizerhof. A systematic development of 'solid-shell' element formulations for linear and non-linear analysis employing only displacement degrees of freedom. International Journal for Numerical Methods in Engineering, 42:49-69, 1998.

[15] K. Knebel. Stabilität von Stahlzylindern mit unilateralen Randbedingungen bei statischen und dynamischen Beanspruchungen. PhD thesis, Institut für Mechanik der Universität Karlsruhe (TH), 1997. 
[16] H. J.-P. Morand and R. Ohayon. Fluid Structure Interaction. John Wiley \& Sons, 1995.

[17] S. Pissanetsky. Sparse matrix technology. Academic Press, 1984.

[18] T. Rumpel. Effiziente Diskretisierung von statischen Fluid-Struktur-Problemen bei großen Deformationen. PhD thesis, Institut für Mechanik der Universität Karlsruhe (TH), 2003.

[19] T. Rumpel and K. Schweizerhof. Volume-dependent pressure loading and its influence on the stability of structures. International Journal for Numerical Methods in Engineering, 56:211-238, 2003.

[20] T. Rumpel and K. Schweizerhof. Hydrostatic fluid loading in non-linear finite element analysis. International Journal for Numerical Methods in Engineering, 59:849-870, 2004 .

[21] T. Rumpel, K. Schweizerhof, and M. Haßler. Efficient finite element modelling and simulation of gas and fluid supported membrane and shell structures. Recent Advances in Textile Membranes and Inflatable Structures, E. Onate, B. Kröplin (eds.), 2004.

[22] J. Schotte and R. Ohayon. Effect of gravity on a free-free elastic tank partially filled with incompressible liquid. Journal of Fluids and Structures, 18:215-226, 2003.

[23] J. Schotte and R. Ohayon. Incompressible hydroelastic vibrations: finite element modelling of the elastogravity operator. Computers and Structures, 83:209-219, 2005.

[24] K. Schweizerhof. Nichtlineare Berechnung von Tragwerken unter verformungsabhängiger Belastung. PhD thesis, Institut für Baustatik der Universität Stuttgart, 1982.

[25] K. Schweizerhof. Quasi-Newton Verfahren und Kurvenverfolgungsalgorithmen für die Lösung nichtlinearer Gleichungssysteme in der Strukturmechanik. Institut für Baustatik der Universität Karlsruhe (TH), 1989.

[26] K. Schweizerhof and E. Ramm. Displacement dependent pressure loads in non-linear finite element analyses. Computers and Structures, 18:1099-1114, 1984.

[27] P. Wriggers. Nichtlineare Finite-Element-Methoden. Springer-Verlag Berlin, 2001.

[28] H. Ziegler. An introduction to thermomechanics. North-Holland Publishing Company, 1977.

[29] O. C. Zienkiewicz and R. L. Taylor. The finite element method, Vol. 1. ButterwothHeinemann, 2000. 